\title{
Baroclinic and barotropic instabilities in planetary atmospheres: energetics, equilibration and adjustment
}

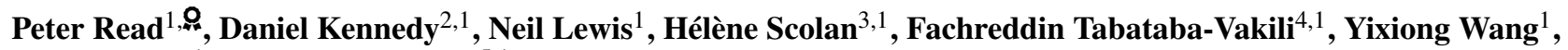 \\ Susie Wright ${ }^{1}$, and Roland Young ${ }^{5,1}$ \\ ${ }^{1}$ Clarendon Laboratory, Department of Physics, University of Oxford, Parks Road, Oxford, OX1 3PU, UK \\ ${ }^{2}$ Max-Planck-Institut für Plasmaphysik, Greifswald, Germany \\ ${ }^{3}$ Laboratoire de Mécanique des Fluides et d'Acoustique, Université de Lyon, France \\ ${ }^{4}$ Jet Propulsion Laboratory, Pasadena, California, USA \\ ${ }^{5}$ Department of Physics \& National Space Science and Technology Center, United Arab Emirates University, Al Ain, United \\ Arab Emirates \\ Invited contribution by Peter Read, recipient of the EGU Lewis Fry Richardson Medal 2016.
}

Correspondence: Peter Read (peter.read@physics.ox.ac.uk)

Received: 3 October 2019 - Discussion started: 15 October 2019

Revised: 14 February 2020 - Accepted: 26 February 2020 - Published: 3 April 2020

\begin{abstract}
Baroclinic and barotropic instabilities are well known as the mechanisms responsible for the production of the dominant energy-containing eddies in the atmospheres of Earth and several other planets, as well as Earth's oceans. Here we consider insights provided by both linear and nonlinear instability theories into the conditions under which such instabilities may occur, with reference to forced and dissipative flows obtainable in the laboratory, in simplified numerical atmospheric circulation models and in the planets of our solar system. The equilibration of such instabilities is also of great importance in understanding the structure and energetics of the observable circulation of atmospheres and oceans. Various ideas have been proposed concerning the ways in which baroclinic and barotropic instabilities grow to a large amplitude and saturate whilst also modifying their background flow and environment. This remains an area that continues to challenge theoreticians and observers, though some progress has been made. The notion that such instabilities may act under some conditions to adjust the background flow towards a critical state is explored here in the context of both laboratory systems and planetary atmospheres. Evidence for such adjustment processes is found relating to baroclinic instabilities under a range of conditions where the efficiency of eddy and zonal-mean heat transport may mutually compensate in maintaining a nearly invariant thermal structure in the zonal mean. In other systems, barotropic in-
\end{abstract}

stabilities may efficiently mix potential vorticity to result in a flow configuration that is found to approach a marginally unstable state with respect to Arnol'd's second stability theorem. We discuss the implications of these findings and identify some outstanding open questions.

\section{Introduction}

One of the great achievements of the past 100 years in fluid dynamics has been the development of a theory of dynamical instability, both linear and nonlinear. In a geophysical context, this has led to a quantitative understanding of a variety of phenomena, including the processes that lead to the development of large-scale energetic eddies in rotating, stratified atmospheres and oceans. These instabilities, known as baroclinic and barotropic instabilities, are well known as the mechanisms responsible for the production of the dominant energy-containing eddies in the atmospheres of Earth and several other planets, as well as Earth's oceans. In general they occur in flows for which background rotation plays an important role, while baroclinic instabilities also require statically stable stratification. They are typically distinguished energetically by the respective dominance of exchanges of either kinetic or potential energy with the background (usually zonal) flow. Barotropic instability is associated with di- 
rect exchanges of kinetic energy between background flow and eddies, while baroclinic instability entails the growth of eddy kinetic and potential energy at the primary expense of the potential energy of the background flow.

These exchanges of energy between zonally symmetric flows and eddies and between potential and kinetic energies are commonly quantified in the manner originally suggested by Lorenz $(1955,1967)$, building on the much earlier work of Margules (1903), in which the flow is partitioned between zonally symmetric (zonally averaged) components and departures therefrom ("eddies"), with four main energy reservoirs (AE, $\mathrm{AZ}, \mathrm{KE}$ and $\mathrm{KZ}$ ) representing respectively the eddy and zonal available potential energy and the corresponding kinetic energies. From considerations of the energy conservation equations, expressions can be defined to represent the rates of conversion between the four energy reservoirs (e.g. see Lorenz, 1967; Peixoto and Oort, 1974; James, 1994), typically shown schematically in Fig. 1a.

As will be discussed further below, baroclinic or barotropic instabilities and axisymmetric overturning circulations can be distinguished energetically by differing routes of energy conversion between potential and kinetic energies. But schematically, barotropic instabilities are characterised by the dominance of the CK conversion term from zonalmean kinetic energy KZ to eddy KE (see Fig. 1c), while energy conversions in baroclinic instabilities are dominated by the $\mathrm{CA}$ and $\mathrm{CE}$ terms, converting zonal-mean potential energy AZ to eddy potential and kinetic energy AE and KE (see Fig. 1d). The direct kinetic energy conversion between $\mathrm{KE}$ and $\mathrm{KZ}$ may also take place in the latter case in either direction and so is indicated by the dashed arrows in Fig. 1d. Such circulations may also be accompanied by direct, axisymmetric overturning circulations that primarily involve the $\mathrm{CZ}$ conversion between $\mathrm{AZ}$ and $\mathrm{KZ}$ (e.g. see Fig. 1b).

In addition to straightforward energy considerations, however, the vorticity configuration (either absolute or potential vorticity) also plays a key role in determining whether an instability develops and the character of the instability when it occurs. The subsequent nonlinear development of the instability then depends also on both energetic and vorticity constraints, posing significant challenges to our understanding of how such instabilities will equilibrate.

Predicting and quantifying such equilibration processes are crucial tasks if we are to gain a full understanding of the factors that govern the observable structure and evolution of atmospheric circulation systems under a variety of different conditions. Recent exploration of our solar system has already provided much information on the structure and properties of large- and medium-scale circulation systems across a wide range of parameter space, from very slowly rotating terrestrial planets such as Venus and Titan through rapidly rotating terrestrial planets such as Earth and Mars to the rapidly rotating gas and ice giants. Baroclinic and/or barotropic instabilities likely play important roles in most of these atmospheric systems, but precisely how the occurrence and nonlinear development of such instabilities governs the structure and energetics of their circulation is still very much the subject of ongoing research, using both observations and numerical circulation models. Many planetary atmospheric circulation systems, for example, manifest complex, often anisotropic and inhomogeneous macroturbulent cascades of energy and enstrophy (squared vorticity), but how are such cascades energised, and what processes determine the typical equilibrated distribution of energy? How are such states maintained by the input of thermal energy from the Sun or from the deep planetary interior?

Determining the occurrence or otherwise of either barotropic or baroclinic instabilities has traditionally appealed to the results of linearised theories, often (though not exclusively) derived from simplified forms of the equations of motion, continuity and energy conservation. The most well known approach derives from the Charney-SternPedlosky (CSP) criterion for the existence of a non-zero growth rate of an infinitesimal perturbation to a background zonal flow (e.g. Vallis, 2017). This essentially reduces to the requirement that

$$
-c_{\mathrm{i}} \int_{y_{1}}^{y_{2}}\left\{\int_{0}^{H} \frac{\partial Q / \partial y}{\left|U-c_{\mathrm{r}}\right|^{2}}|\tilde{\psi}|^{2} \mathrm{~d} z+\left[\frac{F \partial U / \partial z}{\left|U-c_{\mathrm{r}}\right|^{2}}|\tilde{\psi}|^{2}\right]_{0}^{H}\right\} \mathrm{d} y=0,
$$

where $U(y, z)$ is the background zonal flow, $Q(y, z)$ is the corresponding quasi-geostrophic potential vorticity (QGPV), $y$ is the lateral and $z$ is the vertical coordinate, $c=c_{\mathrm{r}}+i c_{\mathrm{i}}$ is the complex phase speed of the perturbation, $\tilde{\psi}(y, z)$ is the amplitude of the perturbation streamfunction, and $F=$ $f_{0}^{2} / N^{2} . f_{0}=2 \Omega \sin \phi_{0}$ is the mean Coriolis parameter, centred at latitude $\phi_{0}$ with $\Omega$ the planetary rotation rate, and $N$ is the Brunt-Väisälä frequency. The integrals are carried out over a domain in $(y, z)$ bounded by rigid walls or other suitable boundary conditions at $y=y_{1}, y_{2}$ and $z=0, H$.

For $c_{\mathrm{i}} \neq 0$, therefore, we require one or more of the following criteria to be satisfied:

(i) $\partial Q / \partial y$ changes sign in the interior domain;

(ii) the interior $\partial Q / \partial y$ take the opposite sign to $\partial U / \partial z$ at the upper boundary at $z=H$;

(iii) the interior $\partial Q / \partial y$ take the same sign as $\partial U / \partial z$ at the lower boundary at $z=0$; or

(iv) $\partial Q / \partial y=0$ in the interior, and $\partial U / \partial z$ takes the same sign at both $z=0$ and $z=H$.

Thus, Earth's atmosphere typically satisfies criterion (iii), being dominated by the planetary vorticity gradient $\beta=\mathrm{d} f / \mathrm{d} y$ away from the lower boundary and $\partial U / \partial z>0$ at the ground, consistent with geostrophic balance and an equatorward temperature gradient. In the oceans, in contrast, either criteria (i) 


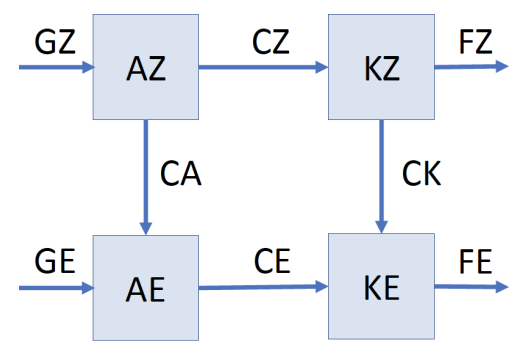

(a)

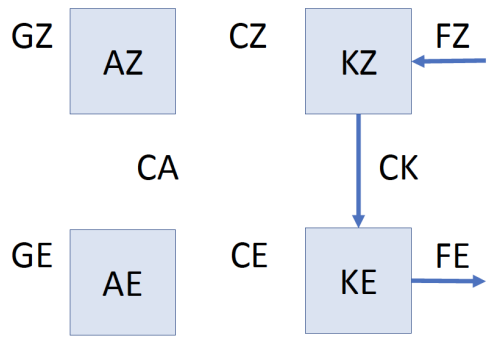

(c)

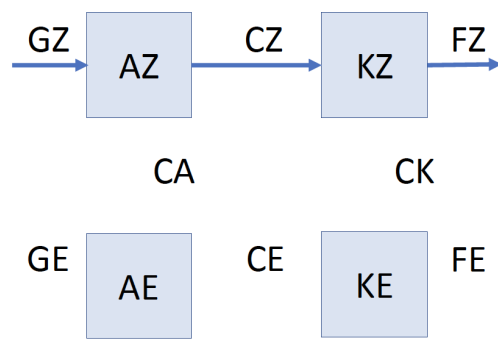

(b)

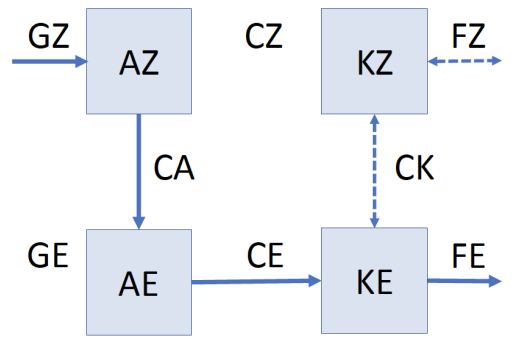

(d)

Figure 1. (a) Schematic representation of energy storage and interconversions in the form suggested by Lorenz (1967), with each box representing the energy reservoirs for eddy and zonal-mean kinetic (KE and KZ) and available potential energies (AE and AZ) and arrows representing conversion rates between them $(\mathrm{CZ}, \mathrm{CK}, \mathrm{CE}$ and CA). Sources or sinks of potential or kinetic energy are also shown as external arrows (GZ, GE, FZ and FE). (b)-(d) Idealised examples representing the cycles expected for isolated processes (e.g. see James, 1994): (b) thermally direct Hadley cell, (c) barotropic instabilities and (d) baroclinic instabilities.

or (ii) may be satisfied to result in mesoscale eddies generated by baroclinic instability. But whether or how these instability criteria may be satisfied on other planets, where conditions may be very different from Earth, is often unclear, leaving open questions as to the respective roles of baroclinic or barotropic instability in the global circulation.

Even where it can be established that one or another CSP criterion can be satisfied, the fully developed nonlinear form of the instability may also be hard to predict without the use of complex and expensive numerical models. One possible hypothesis, however, that has proved insightful and interesting in some circumstances is the suggestion that the instability grows vigorously and feeds back onto the background flow, tending to restore it to a state of marginal instability or some other well-defined state. For baroclinic instabilities, this process is often referred to as "baroclinic adjustment" (e.g. Stone, 1978; Zurita-Gotor and Lindzen, 2007; Schneider, 2007) by analogy with the concept of rapid convective adjustment towards a state of neutral static stability in a convectively unstable flow. Such a process might be expected to occur where the background unstable flow is only weakly forced or maintained, on timescales that are long compared with the advective timescale of the developing baroclinic instability. The applicability of this concept to Earth's atmosphere was first suggested by Stone (1978), based on an application of the Phillips two-layer model of baroclinic instability. However, the validity of this application is controver- sial because Earth's atmosphere is always baroclinically unstable, even though the dominant perturbations may become much shallower than the height of the troposphere. Thus, a state of marginal instability is not well defined, leading to alternative hypotheses for the adjusted state.

In this paper we consider the application of the CSP and other stability criteria to rotating, stratified flows that include laboratory experiments and both idealised and observed planetary atmospheres. We also explore some of the properties of the fully developed circulations with a view to quantifying the energetics of the equilibrated state and identifying the action and consequences of either baroclinic or barotropic adjustment. Section 2 reviews the results of laboratory experiments on fully developed baroclinic and barotropic instabilities across a broad range of parameter space, examining the usefulness or otherwise of the CSP criteria and any evidence for signatures of adjustment or other forms of self-organised criticality. Section 3 reviews the corresponding properties of idealised general circulation model (GCM) simulations of an Earth-like planetary atmosphere circulation over a broad range of rotation rates. This is for comparison in Sect. 4 with the known properties of the atmospheric circulations of the main planetary bodies of the solar system. We draw some conclusions and an outlook for future research in Sect. 5. 


\section{Baroclinic and barotropic instabilities in the laboratory}

Laboratory experiments on rotating, stratified flows provide a powerful vehicle for exploring and understanding the fully developed forms of processes such as baroclinic and barotropic instability. The imposed boundary conditions and experimental parameters can often be closely controlled so that experiments can be repeated and checked, while the geometry can be kept relatively simple so that the respective influence of different factors can be tested over a wide range of conditions. Measurement techniques have also advanced considerably in recent years, allowing for optical and other non-intrusive flow measurements as well as the use of in situ probes. Both baroclinic and barotropic flow configurations have been explored in past work over the past 50 or more years, with insightful results on bifurcations between steady, periodic and chaotic flow states and heat and momentum transfer properties.

\subsection{Barotropic instabilities of shear layers and jets}

\subsubsection{CSP stability criteria and energy exchanges}

For a purely barotropic flow, the CSP analysis reduces $\partial Q / \partial y$ to $\beta-\partial^{2} U / \partial y^{2}$, leading to stability criterion (i) taking the familiar Rayleigh-Kuo form for the stability of a two-dimensional flow. In effect this represents the need for an inflection point (or related feature) in a zonal shear flow as a necessary but insufficient criterion for its instability. Such a criterion is necessary to enable the possibility of supporting a pair of zonally propagating Rossby-like waves that can phase-lock across the inflection point and lead to the growth of an instability e.g. via over-reflection (e.g. see Lindzen, 1988). The combination of local vorticity gradients and Doppler shifting of Rossby-like wave trains located in different parts of the flow allow for the possibility of pairs of such wave trains to propagate at the same velocity relative to their common frame of reference. Provided that their lateral extent is sufficient for them to interact, these pairs of wave trains can remain mutually coherent and stationary in a common frame of reference moving with the waves, allowing them to interact strongly and draw kinetic energy from the background flow if their phase relationship is favourable.

This generally entails a phase tilt across the channel in such a way that the waves "lean into" the sheared zonal flow so that the momentum flux $\overline{u^{*} v^{*}}$ acts to reduce the shear of the background zonal flow. This correlation between the eddy momentum flux and zonal shear is quantified by the CK term in the Lorenz energy budget,

$\mathrm{CK}=\left\langle\frac{\mathrm{d} \overline{[u]}}{\mathrm{d} y} \overline{\left[u^{*} v^{*}\right]}\right\rangle$,

where $u^{*}$ and $v^{*}$ are perturbation velocities with respect to the zonal mean, indicated by the overbar, angle brackets de- note a vertically integrated areal average and square brackets denote a time average. This leads naturally to $\mathrm{CK}>0$ such that kinetic energy is transferred from $\mathrm{KZ}$ to $\mathrm{KE}$.

\subsubsection{Barotropically unstable flows in the laboratory}

It turns out that generating a flow in the laboratory with such an inflection point favourable for supporting phaselocked Rossby wave pairs is reasonably straightforward because such a flow structure occurs spontaneously in a viscous Stewartson shear layer. A detached shear layer may be produced in a rotating, homogeneous fluid by the use of differentially rotating horizontal boundaries, e.g. by the use of a differentially rotating disk or ring at the centre of a cylindrical tank. This was originally investigated by Hide and Titman (1967) and later explored in experiments by Früh and Read (1999a), Früh and Read (1999b), and Aguiar et al. (2010). A schematic diagram of the apparatus is shown in Fig. 2a-b, and a typical apparatus is illustrated in Fig. 2c.

The resulting directly forced zonal flow has a clear inflection point in its radial velocity profile in such a way that the radial vorticity gradient clearly changes sign. A typical example is illustrated in Fig. 3 for a jet-like flow, driven by a differentially rotating ring. The azimuthal velocity is shown in Fig. 3a, while the corresponding vorticity gradient is shown in Fig. $3 b$.

This would appear to suggest that the flow is unstable, yet it is observed in this state in a time average under conditions in which a regular azimuthal wavenumber $m=6$ flow is found. The CSP condition, however, is only a necessary condition for instability, not a sufficient one. An alternative condition for instability was considered by Arnol'd (1966) and is often referenced as "Arnol'd's second stability theorem" (e.g. Dowling, 1995). This approach considers the instability that could arise from the interaction of two (or more) parallel wave trains (commonly, as herein, taken to be Rossby waves, although this may be generalised to other interacting wave modes; e.g. see Ripa, 1983; Hayashi and Young, 1997; Sakai, 1989; Gula et al., 2009; Flór et al., 2011), each centred on a local maximum or minimum of the potential vorticity gradient. The existence or otherwise of a steadily moving reference frame in which both wave trains, Doppler shifted by the zonal flows in their vicinity, can be at rest relative to each other provides a criterion for their sustained mutual interaction and hence exchange of momentum to gain energy at the expense of the background zonal flow. The resulting condition for perturbations to be stable is of the form

$\left(\frac{\bar{u}-\alpha}{\partial \bar{Q} / \partial y}\right) \geq L_{0}^{2}$,

where $L_{0}$ is a constant length scale which, depending on the model, may represent the Rossby radius of deformation $\left(L_{\mathrm{D}}\right)$ and $\alpha$ is a constant representing the speed of the waves in a shifted inertial reference frame. The marginal stability condition is set by replacing the inequality in Eq. (3) with an 


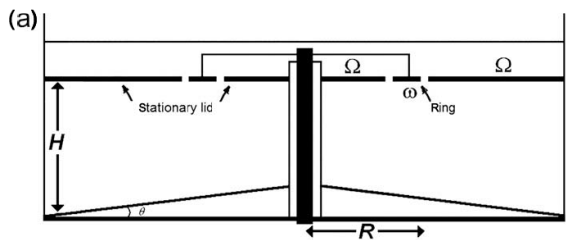

(b)
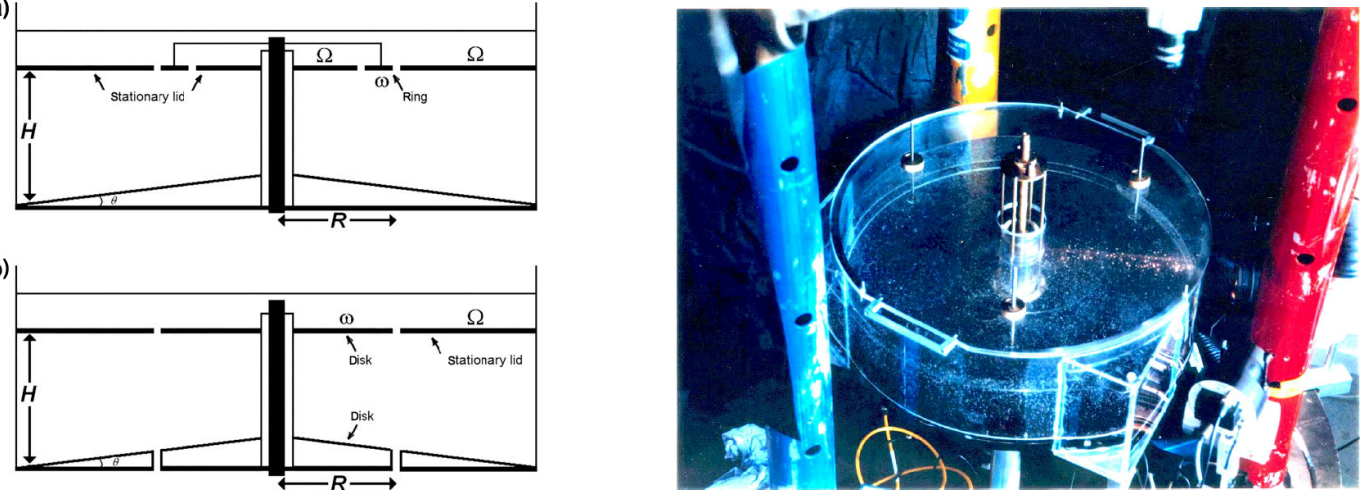

Figure 2. Schematic cross sections of two versions of the apparatus for producing and studying barotropic instabilities in a cylindrical tank of fluid on a rotating table at angular velocity $\Omega$. In (a) a differentially rotating, axisymmetric ring is used to drive a barotropic jet at relative angular velocity $\omega$ at radius $R$, while in (b) a pair of differentially rotating disks, centred on the rotation axis, generate a detached Stewartson shear layer, also at radius $R$. In these cases, a topographic $\beta$ effect is also included by the use of a conically sloping bottom with slope angle $\theta$. (c) Photograph of the detached shear layer apparatus as used by Früh and Read (1999a) for investigating fully developed barotropic instabilities.

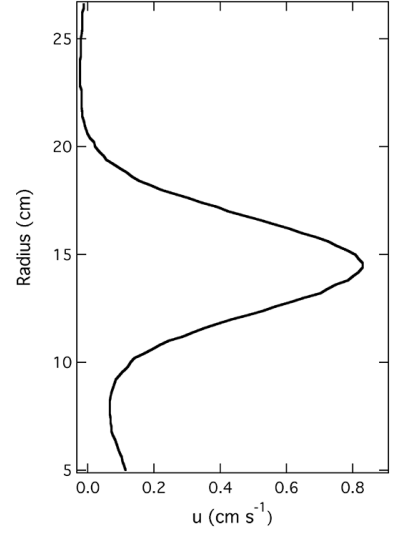

(a)

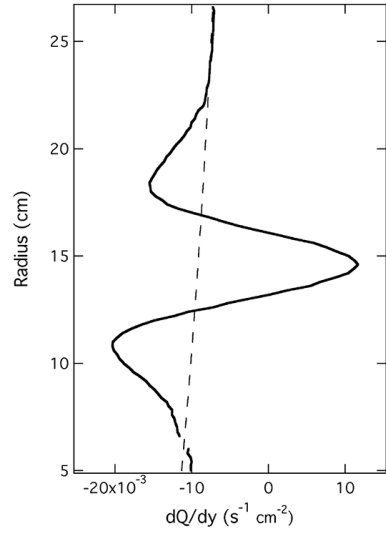

(b)

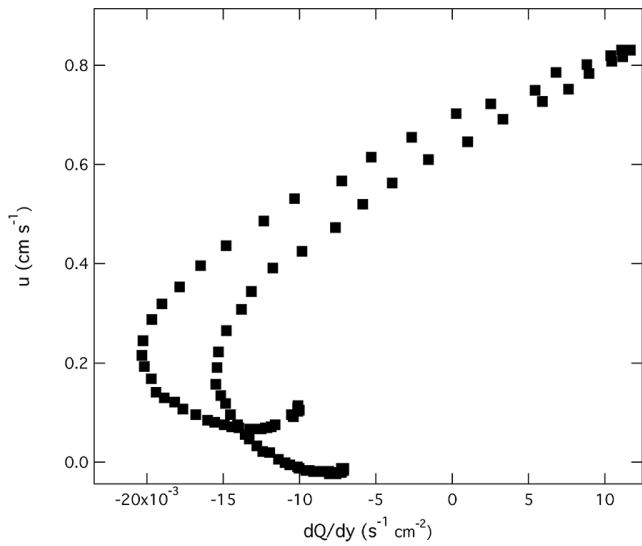

(c)

Figure 3. A typical azimuthal and time mean jet flow, driven by a differentially rotating ring as used by Aguiar et al. (2010), derived from particle image velocimetry measurements. Panel (a) shows the radial profile of azimuthal velocity $(u)$, while panel (b) shows the corresponding radial gradient of shallow-water potential vorticity, clearly confirming a change of sign of $\partial Q / \partial y=-\partial Q / \partial r$ at $r \simeq 12$ and $17 \mathrm{~cm}$, on either side of the barotropic jet ( $\partial Q / \partial y$ due solely to the gradient of $2 \Omega / h$ shown as a dashed line). Panel (c) shows a scatter plot of $u$ against $\partial Q / \partial y$, illustrating the nearly linear relationship between the two quantities within the barotropically unstable jet for $\bar{u}>0.2 \mathrm{~cm} \mathrm{~s}^{-1}$.

equality such that $\bar{u}$ is linearly related to $\partial \bar{Q} / \partial y$ with an offset $\alpha$ at the intercept where $\partial \bar{Q} / \partial y=0$ (e.g. Dowling, 1995).

In practice, however, marginally stable real flows with both forcing and dissipation will not obey this condition exactly but may approximate to it if the forcing and dissipation are relatively weak compared to the advective transport of $Q$. For the laboratory flow presented in Fig. $3 a$ and $b$, this is illustrated in Fig. 3c, which shows a plot of $\bar{u}$ against $-\partial \bar{Q} / \partial r$ ( $=\partial \bar{Q} / \partial y$ with $y$ directed towards the rotation axis), where $Q$ is now the shallow-water potential vorticity

$Q=\frac{\zeta+2 \Omega}{h(r)}$, $\zeta$ is the relative vorticity and $h(r)$ is the radius-dependent depth of the fluid layer. Figure $3 \mathrm{c}$ clearly shows a strong and nearly linear correlation between $\partial \bar{Q} / \partial y$ and $\bar{u}$ where $\bar{u}>0.2 \mathrm{~cm} \mathrm{~s}^{-1}$, corresponding to the core of the zonal jet in Fig. 3a. Thus, even though the equilibrated time-averaged flow continues to satisfy the CSP condition for instability, it has effectively stabilised the flow to a configuration that is close to marginal stability with regard to Arnol'd's second stability condition (hereafter "Arnol'd II"), as a result of a process which can be regarded as a form of barotropic adjustment. 


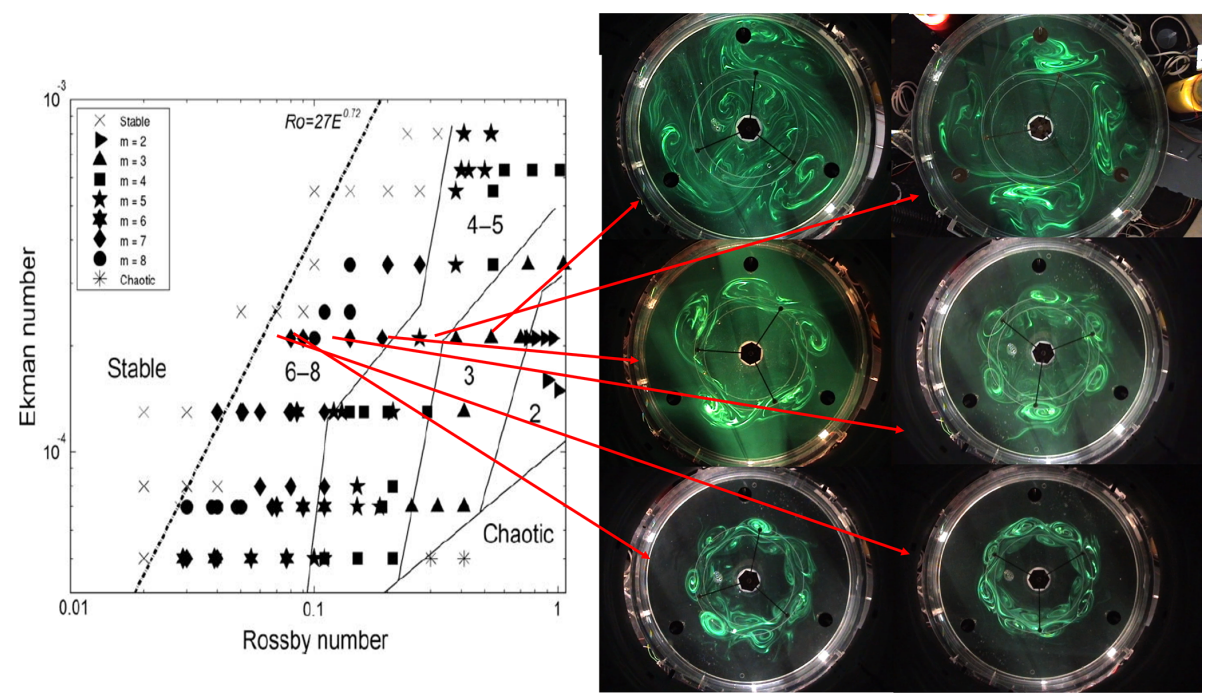

Figure 4. Regime diagram for the barotropic jet flows as a function of the Rossby and Ekman number, as obtained by Aguiar et al. (2010), showing snapshots of typical equilibrated flows as visualised using dye and side illumination. The trend towards a higher wavenumber, at the stability boundary as $R e=R e_{\mathrm{c}}$, is approached is clearly seen.

\subsubsection{Circulation regimes and wavenumber selection}

The resulting flows thus equilibrate to a marginally stable state in which the original jet meanders in the form of a train of azimuthally travelling waves, on either side of which are patterns of closed vortices whose vorticity matches in sign with that of the flanks of the zonal jet. The flow is typically found to select just one dominant zonal wavenumber in many of the published experiments (e.g. see Fig. 4), the wavelength of which depends mainly upon a combination of the Rossby $R o$ and Ekman $E$ numbers, commonly defined (e.g. Früh and Read, 1999a, b) as

$$
\begin{aligned}
& R o=\frac{R \omega}{2 \bar{\Omega} d}, \\
& E=\frac{v}{\bar{\Omega} d^{2}},
\end{aligned}
$$

where $R$ is the radius and $\omega$ is the relative angular velocity of the shear layer or jet, $v$ is the kinematic viscosity of the fluid, and $d$ is the depth of the fluid layer. $\bar{\Omega}$ is the mean angular velocity of the fluid, representing the average of the turntable rotation rate $\Omega$ and the differential rotation rate $\omega$.

As shown by Niino and Misawa (1984) and Früh and Read (1999a), although the inviscid CSP criterion (i) or (more realistically) Arnol'd II criterion needs to be satisfied for the driven jet to be unstable, the observed stability boundary in a real, viscous fluid is also consistent with the existence of a critical Reynolds number $R e_{\mathrm{c}}$, defined with respect to a length scale of the same order as that of the $E^{1 / 4}$ Stewartson layer such that

$R e_{\mathrm{c}}=\frac{U L_{\mathrm{S}}}{v} \simeq R o \cdot E^{-3 / 4} \simeq 27$, where

$$
L_{\mathrm{S}}=(E / 4)^{1 / 4} d
$$

is the width of the viscous Stewartson layer. This expression provides a reasonably accurate description of the observed stability boundary (e.g. see Fig. 4) for both the jet and detached shear layer flows, such that for $R e<R e_{\mathrm{c}}$ the flow observed is essentially axisymmetric with no discernible wave perturbation.

The corresponding boundaries between different flow regimes dominated by different wavenumbers are observed empirically to lie almost parallel to the main stability boundary, suggesting that the wavenumber selected is largely determined by the effective Reynolds number and hence the effective supercriticality of the barotropic instability $\left(R e-R e_{\mathrm{c}}\right)$. In fully developed flows this may be consistent with other work on scale selection in barotropic instability, which associates the most unstable wavelength with a scale comparable to the width of the background jet or shear layer (e.g. Sommeria et al., 1991; Vallis, 2017). In these barotropic instability experiments, however, the equilibrated amplitude of unstable waves grows with supercriticality, effectively broadening the width of the original jet beyond its initial Stewartson layer width. At its equilibrated finite amplitude, therefore, strongly supercritical flows will tend to favour longer wavelength disturbances, qualitatively consistent with the observed ordering of different wavenumber regimes. This can be clearly seen in Fig. 4, in which the lower wavenumber states are seen to fill the entire tank with large meanders and vortices, whereas high wavenumber flows tend to be more tightly confined in radius to the vicinity of the unstable jet beneath the rotating ring. At large supercritical Reynolds numbers, however, 
the equilibrated flow may no longer be steady but may become chaotic, as observed at the lowest Ekman numbers (see Fig. 4), although this has not been explored in much detail as of yet.

This method of forcing and maintaining a barotropically unstable flow is relatively strong and fast, so even at large equilibrated amplitudes the zonally averaged flow maintains a reversal in the lateral gradient of potential vorticity. The forcing therefore would seem to be too strong to allow the zonally symmetric component of the flow to maintain itself close to a marginally unstable state. The resulting equilibrated flow is therefore strongly nonlinear, especially at relatively large effective Reynolds numbers.

\subsection{Baroclinic instabilities}

Baroclinic instabilities require the maintenance of a zonally symmetric distribution of density or temperature that is statically stable in the vertical direction together with a horizontal temperature gradient. This configuration has been studied intensively for more than 60 years in the laboratory using the thermally driven, rotating annulus (see Fig. 5). A fluid is contained between two upright, coaxial metal cylinders, which can be maintained at two different temperatures. Most typically, the inner cylinder is maintained at a cooler temperature than the outer, schematically representing the thermal contrast maintained between the equator and poles of an Earthlike planetary atmosphere. The annular tank is mounted on a rotating table which rotates at angular velocity $\Omega$, with the axis of rotation aligned with the axis of symmetry of the cylinders, again by analogy with a planetary atmosphere. The thermal contrast $\Delta T$ between the cylinders drives an axisymmetric overturning circulation in the annular channel, mainly confined to boundary layers, which enables a stable stratification to develop and equilibrate, while the effect of background rotation is to induce a vertically sheared azimuthal flow as fluid moves between inner and outer cylinders while roughly conserving its angular momentum.

At a fast enough rotation rate, radial flow becomes largely confined to shallow Ekman layers close to the horizontal (thermally insulating) boundaries, while the isotherms in the interior of the annular channel develop an inclined slope with respect to the horizontal, thus effectively storing potential energy. The flow patterns observed in such a system are then found to depend strongly upon both the rotation rate and the imposed thermal contrast between the cylindrical boundaries (e.g. see Hide and Mason, 1975, and Read et al., 2015, for reviews). The approximate sequence of bifurcations observed between different circulation regimes is shown schematically in Fig. 6 as a function of the principal dimensionless thermal Rossby number $\mathrm{Ro}_{\mathrm{T}}$ and the Taylor number $\mathcal{T}$ defined respectively as

$$
\operatorname{Ro}_{\mathrm{T}}=\frac{g \alpha \Delta T d}{\Omega^{2} L^{2}}
$$

$\mathcal{T}=\frac{4 \Omega^{2} L^{5}}{v^{2} d}$,

where $g$ is the acceleration due to gravity, $\alpha$ is the volumetric expansion coefficient of the fluid, $v$ is the kinematic viscosity, $L$ is the horizontal width of the annular channel and $d$ is its depth.

As is well known, for a given imposed thermal contrast $\Delta T$, the flow is observed to be stable to baroclinic instabilities for rotation rates slower than a critical value (corresponding to $R o_{\mathrm{T}} \gtrsim 2$ ), resulting in an axisymmetric overturning circulation with a prograde flow at upper levels. For higher rotation rates $\left(R o_{\mathrm{T}} \lesssim 2\right)$, the flow becomes unstable to baroclinic disturbances that cause the originally axisymmetric azimuthal flow to meander in the form of a train of azimuthally propagating waves. These baroclinic waves are the fully developed manifestation of baroclinic instability, sometimes referred to as "sloping convection" (e.g. Hide, 1969; Hide and Mason, 1975), and may take the form of either regular, near-monochromatic wave trains or more chaotic or even turbulent flows at the highest rotation rates.

\subsubsection{CSP instability criteria}

Although from an energetic viewpoint it is reasonably clear why the basic state maintained by the differential heating in the rotating annulus experiment can lead to the release of potential energy to energise the growth of wave-like perturbations, it is less clear how the flow might satisfy the CSP criteria for instability. Early work by Hide (1969) compared the conditions under which active sloping convection was observed in the laboratory with various extensions of the linear model of baroclinic instability by Eady (1949). Provided that account was taken of the dissipative effects of viscous Ekman layers adjacent to the upper and lower boundaries, and these comparisons found generally good quantitative agreement for the onset of the instability around a Burger number, defined as

$B u=\frac{N^{2} d^{2}}{4 \Omega^{2} L^{2}}$,

of the order $R o_{\mathrm{T}} / 4 \simeq 0.5$. Such an analogy, however, presumes that potential vorticity gradients in the interior are negligible compared with the thermal gradients at the horizontal boundaries, satisfying the CSP instability condition through criterion (iv).

In practice, however, the strong radial flow within the Ekman layers in typical annulus experiments rapidly transfers hot or cold fluid across the domain, maintaining relatively weak horizontal thermal gradients at the top of each Ekman layer. This would suggest that the potential vorticity dynamics is controlled much less by the structure of the flow close to the horizontal boundaries and is more strongly influenced by a change of sign of $\partial Q / \partial y$ in the interior flow. This would indicate that the basic zonal flow maintained by the differential heating at the side boundaries is closer in character to an 


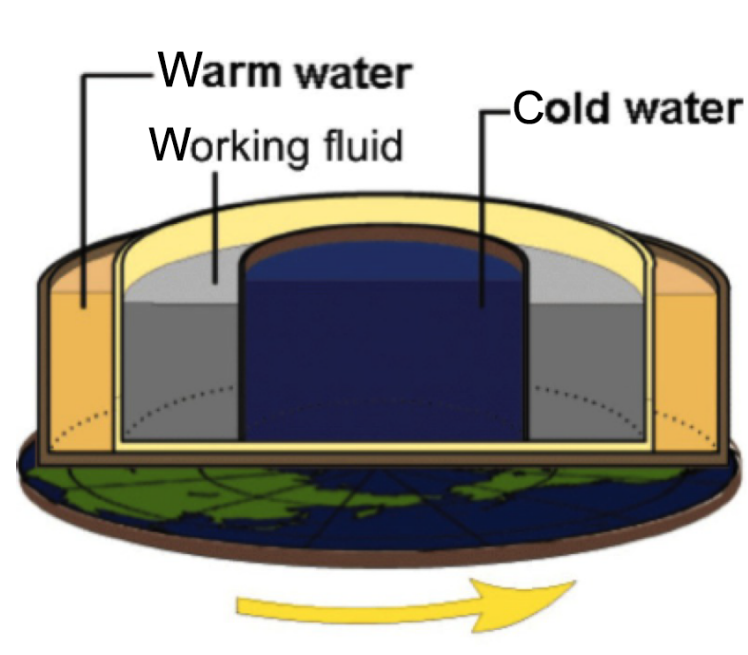

(a)

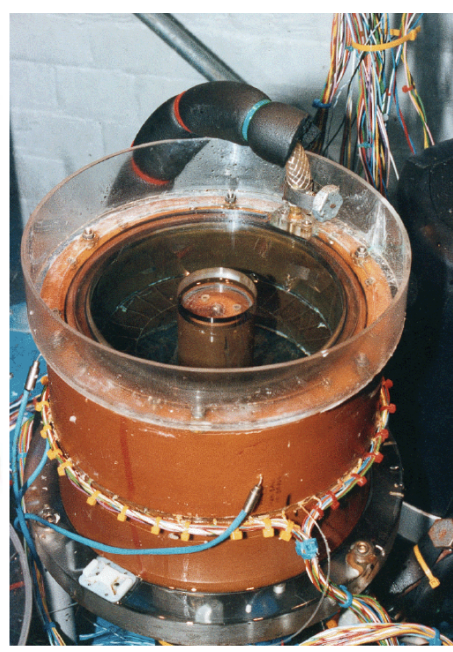

(b)

Figure 5. Schematic layout of the thermally driven, rotating annulus experiment (a) as a vehicle for the study of rotating, stratified flows, with (b) a photograph of a typical apparatus in the laboratory, clearly showing the metallic inner and outer cylinders and connections to heat exchangers for maintaining the temperature of the outer wall and a ring of thermocouple junctions to measure temperatures in the interior.

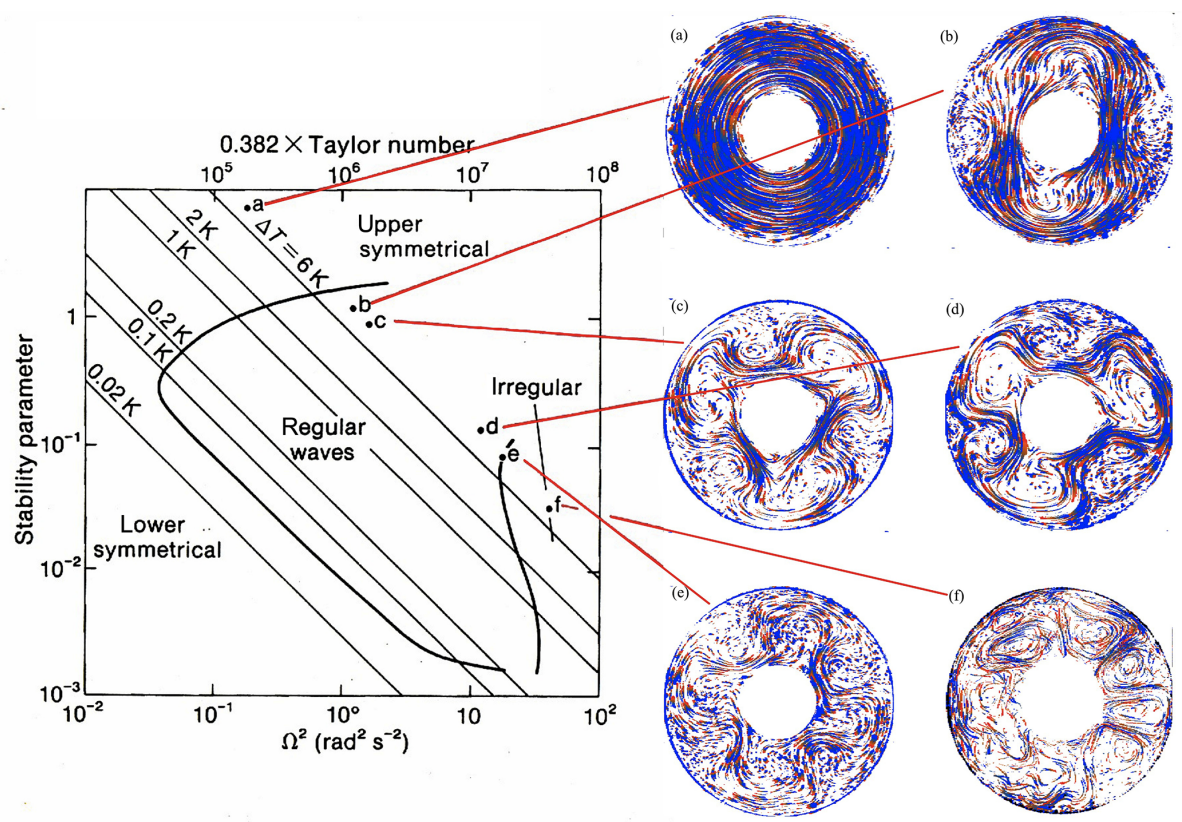

Figure 6. Regime diagram for the baroclinically stable and unstable flows in a thermally driven, rotating annulus as a function of the thermal Rossby number (shown here as "Stability parameter") and Taylor number (e.g. Read et al., 2015), showing snapshots of typical equilibrated flows as visualised using neutrally buoyant tracer particles and side illumination to obtain streak images.

internal jet (e.g. Charney and Stern, 1962; Bell and White, 1988). Baroclinic or barotropic instability would therefore occur through satisfying the CSP conditions directly through criterion (i). The consequences of this in the context of rotating annulus experiments were explored by Bell and White (1988), who noted that idealised, inviscid, baroclinic internal jets without any lateral shear would become unstable at around the same value of the Burger or thermal Rossby number as (or slightly larger than) the classical Eady problem. This was broadly in agreement with experiments, which did show a tendency for the onset of instability to occur at slightly larger values of $R o_{\mathrm{T}}$ than the Eady problem would suggest. However, the critical value of $B u$ or $R o_{\mathrm{T}}$ was found to be quite strongly sensitive to the addition of lateral shear to a baroclinic internal jet (Bell and White, 1988). 


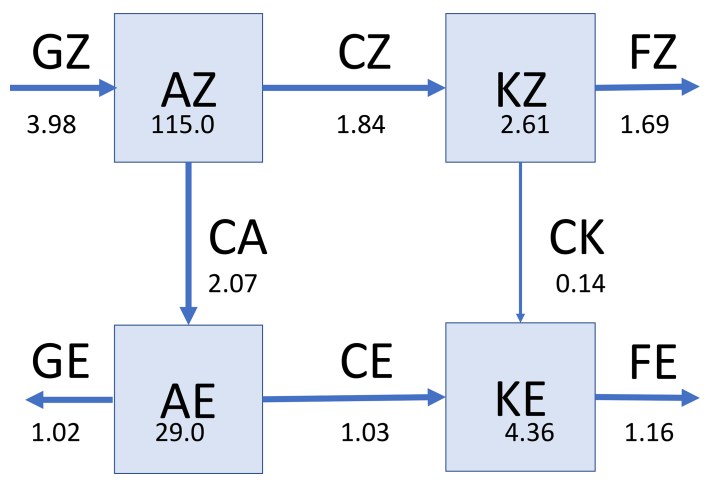

Figure 7. An example Lorenz energy cycle, computed by Young (2014) from a numerical simulation of an equilibrated baroclinic wave flow in a differentially heated, rotating annulus. Energy reservoir and conversion and source/sink terms are in units of $10^{-7}$ joules per kilogram and watt per kilogram respectively.

Numerical analyses, using more realistic linearised models based on the full Boussinesq Navier-Stokes equations with molecular viscosity and thermal diffusion (e.g. Lewis and Nagata, 2004; Lewis et al., 2015, and references therein), however, show close agreement with experimental measurements and also reveal sensitivity of the instability to other factors such as the Prandtl number, especially at low values of both $R_{\mathrm{T}}$ and $\mathcal{T}$ along the boundary of the so-called "lower-symmetric" regime. At a high Prandtl number, the boundary roughly follows a line of constant $\Delta T$, indicative of a critical Rayleigh $\left(R a_{\mathrm{c}}\right)$ or Grashof number $\left(G r_{\mathrm{c}}\right)$ in a similar way to the criterion for Rayleigh-Bénard convection. The product $R o_{\mathrm{T}} \cdot \mathcal{T}$ actually has the form of a Grashof number such that $R o_{\mathrm{T}} \cdot \mathcal{T}=G r_{\mathrm{c}}$ is consistent with the shape of the lower-left instability boundary shown in Fig. 6.

\subsubsection{Lorenz energy cycle}

As instabilities develop and equilibrate, azimuthally travelling waves emerge with characteristic three-dimensional structures and azimuthal phase tilts in radius and height. These result in eddy fluxes of heat and momentum that lead to energy conversions between potential and kinetic energies in the zonally averaged and perturbation fields. A typical example of the Lorenz energy cycle for an equilibrated baroclinic wave developed from a baroclinically unstable initial state is shown in Fig. 7. This was computed by Young (2014) from a numerical simulation of flow in a rotating annulus experiment from solutions of the Boussinesq Navier-Stokes equations in cylindrical annular geometry. The boundary conditions represent non-slip, rigid sidewalls and horizontal end walls, thermally insulating end walls, and isothermal sidewalls at temperatures $T_{\mathrm{a}}$ and $T_{\mathrm{b}}$ at inner and outer radii $r_{\mathrm{a}}$ and $r_{\mathrm{b}}$, with $\Delta T=T_{\mathrm{b}}-T_{\mathrm{a}}=4 \mathrm{~K}, r_{\mathrm{a}}=2.5 \mathrm{~cm}, r_{\mathrm{b}}=8.0 \mathrm{~cm}$, $d=14 \mathrm{~cm}$ and rotation rate $\Omega=3.0 \mathrm{rad} \mathrm{s}^{-1}$, corresponding to $R o_{\mathrm{T}}=0.056$.

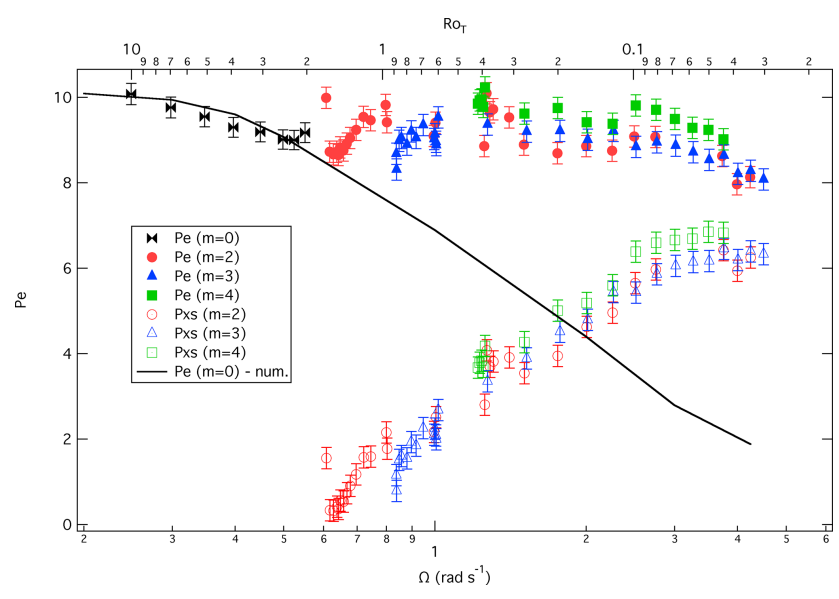

Figure 8. Variations of radial heat transport by baroclinic eddies and axisymmetric flows in a differentially heated rotating annulus, as measured in the laboratory (Read, 2003) as a function of rotation rate and the thermal Rossby number $R o_{\mathrm{T}}$. The total advective heat transport is indicated by the dimensionless Péclet number ( $P e=N u-1$, where $N u$ is Nusselt number; see Eq. 12). Solid symbols represent total heat transport as measured by calorimetry, while the continuous line represents the total heat transport by the axisymmetric flow alone. Open symbols indicate the difference, Pxs, in Pe between measured total advective heat transport and the axisymmetric component, representing the heat transport due to eddies alone of dominant azimuthal wavenumber $m$. Numerical simulation: num.

The values shown in Fig. 7 clearly indicate that the conversion terms implicated in energising baroclinic instability (CA and CE; see Fig. 1b) are dominant in the exchanges between eddies and the zonal-mean flow. $\mathrm{CK}$ is at least a factor of 10 smaller than CA though in the same sense for the generation of KE, indicating that the dominant instability has a somewhat mixed baroclinic-barotropic character. The direct zonal-mean exchanges $(\mathrm{CZ})$ are also of a similar magnitude to $\mathrm{CA}$ and $\mathrm{CE}$ and are consistent with a thermally direct overturning circulation in parallel with the conversion of available potential energy to KE.

\subsubsection{Signatures of baroclinic adjustment in heat transport?}

As mentioned above, baroclinic adjustment is commonly associated with a tendency for baroclinic instability to equilibrate at a finite amplitude in such a way as to modify the initially unstable basic state towards a less unstable configuration. But it is less clear how this tendency might manifest itself in practice. However, one possible way this might be observable could be in the way heat transport by baroclinic disturbances varies with external parameters as the instability threshold is exceeded.

Figure 8 shows a series of calorimetric measurements of total heat transport in a differentially heating rotating annulus experiment for fixed $\Delta T$ as $\Omega$ is varied (see Read, 2003). In this case, heat transport is non-dimensionalised with re- 
spect to thermal conduction, either by the Nusselt number, $\mathrm{Nu}$, defined by

$N u=\frac{\mathcal{H} \ln \left(r_{\mathrm{b}} / r_{\mathrm{a}}\right)}{2 \pi \kappa \mathrm{d} \rho c_{H} \Delta T}$

where $\mathcal{H}$ is the total heat transport in $\mathrm{W}, \kappa$ is the thermal diffusivity of the working fluid and $c_{H}$ is its specific heat capacity, or by the Péclet number $\mathrm{Pe}=\mathrm{Nu}-1$. Thus the solid symbols in Fig. 8 show little variation with $\Omega$ or $R o_{\mathrm{T}}$ until the highest rotation rates. This corresponds to where the regular wave regime in the laboratory experiments begins to break down towards small-scale irregular flows and geostrophic turbulence. The solid line in Fig. 8, on the other hand, shows the variation of $N u$ for a purely axisymmetric flow, obtained from numerical simulations in which nonaxisymmetric eddies were suppressed (see Read, 2003, for details). This shows that the axisymmetric flow under similar conditions decays much more quickly with $\Omega$ than when baroclinic waves are allowed to develop. The corresponding contribution to the dimensionless total heat transport by baroclinic waves is indicated by the open symbols, which are obtained from the difference between total heat transport and that of the pure axisymmetric flow. This clearly shows that the eddy contribution increases systematically from zero as the rotation rate is increased beyond the critical value for the onset of instability, until it begins to saturate and even begin to turn over for $R_{\mathrm{T}}<0.1$.

The observation that total heat transport may be almost independent of rotation rate over a wide range of $\Omega$, from the axisymmetric regime into the fully developed baroclinic wave regime, has been noted since the early work of Bowden (1961), who was the first to carry out calorimetric measurements of heat transport in rotating annulus experiments. This tendency for the heat transport to remain close to its value at $\Omega=0$, even though the (mainly axisymmetric) boundary layer contribution should reduce substantially over the same range of parameters, is unlikely to be coincidental, but it more likely reflects a systematic result of equilibrated baroclinic instability in modifying the overall flow to transport almost as much heat (and therefore release almost as much potential energy) as the non-rotating flow. The non-rotating flow represents a fully relaxed state, in which isotherms and isopycnals are as nearly horizontal as possible (except in conductive boundary layers) and is therefore a state that would be energetically stable to further baroclinic instabilities. In this sense, these results demonstrate that rotating annulus flows are capable of exhibiting a relatively strong form of baroclinic adjustment throughout the regular baroclinic wave regime. It is only when the regular regime begins itself to become unstable at values of $R o_{\mathrm{T}} \ll 0.1$, where the dominant energetic length scale of the baroclinic waves becomes significantly smaller than the width of the imposed baroclinic zone, that the eddies are no longer able to sustain the level of heat transfer required to maintain the fully relaxed flow state and that the baroclinically adjusted state breaks down to a more strongly supercritical flow.

Such a tendency would seem to be quite general, and so it is of interest to see whether similar trends might be observable in a planetary atmosphere in which differential heating is maintained by (radiative) relaxation towards a baroclinically unstable radiative equilibrium state.

\section{Baroclinic and barotropic instabilities in idealised planetary atmospheric circulations}

Earth and other solar-system planets present us with just a few samples of atmospheric circulation systems in different parts of a broader parameter space (e.g. see Read, 2011). A better way of exploring trends and the scaling of various properties of planetary atmosphere circulations is to use a simplified numerical model, in which the key forcing processes are represented schematically but in a physically consistent manner as a function of the principal planetary parameters so that the model can be used to explore the relevant dynamical response. This approach has a long history, dating back to the early work of Hunt (1979), Williams and Holloway (1982), and Geisler et al. (1983), but has become more common in recent years, inspired in part by the diversity of newly discovered extrasolar planets (e.g. Merlis and Schneider, 2010; Mitchell and Vallis, 2010; Kaspi and Showman, 2015; Wang et al., 2018). Here we focus on the diagnostics of energetics, instabilities and heat transfer in a typical set of idealised GCM simulations, based on the work of Wang et al. (2018).

\subsection{Circulation regimes}

As demonstrated by various authors (e.g. Kaspi and Showman, 2015; Wang et al., 2018), many generic features of the large-scale circulation of almost any planetary atmosphere can be captured in a general circulation numerical model that solves the hydrostatic primitive equations of meteorology, together with simple parameterisations of diabatic heating and small-scale turbulent mixing and friction. In recent work, Wang et al. (2018) has used the University of Hamburg PUMA (Portable University Model of the Atmosphere) model (e.g. Frisius et al., 1998; Fraedrich et al., 2005) to explore the dependence of the simulated fully three-dimensional, time-dependent circulation of an Earthlike planetary atmosphere on dimensionless parameters such as the thermal Rossby number $R o_{\mathrm{T}}$ as quantities such as the planetary rotation rate $\Omega^{*}=\Omega / \Omega_{\mathrm{E}}$ (where $\Omega_{\mathrm{E}}$ is the rotation rate of Earth) are varied. This model represents fields in the horizontal dimension as projections onto sets of spherical harmonic functions but in a finite-difference form in the vertical dimension. Diabatic heating and cooling is represented by a linear relaxation towards a prescribed zonally symmetric temperature field, intended to represent the diurnally and 
seasonally averaged radiative-convective equilibrium of an Earth-like planet, with a prescribed relaxation timescale $\tau_{\mathrm{R}}$. Surface friction is represented by a height-dependent, linear Rayleigh friction parameterisation with local timescale $\tau_{\mathrm{F}}$ and total (Ekman) spin-down timescale of $\tau_{\mathrm{S}}$.

The model was run to equilibrium over 10-20 Earth years before computing various diagnostics of the statistically equilibrated state. The circulation regime could then be characterised as a function of various dimensionless parameters, such as the thermal Rossby number,

$R o_{\mathrm{T}}=\frac{R \Delta \theta_{\mathrm{EP}}}{\Omega^{2} a^{2}}$,

where $R$ is the specific gas constant, $\Delta \theta_{\mathrm{EP}}$ is the horizontal temperature contrast between the equator and poles, and $a$ is the planetary radius, the Burger number,

$B u=\frac{N^{2} H^{2}}{4 \Omega^{2} a^{2}} \simeq \frac{R \Delta \theta_{z}}{4 \Omega^{2} a^{2}}$,

where $\Delta \theta_{z}$ is the vertical contrast in potential temperature and frictional or radiative Taylor numbers,

$\mathcal{T}_{(\mathrm{F}, \mathrm{R})}=4\left(\Omega\left(\tau_{\mathrm{F}}, \tau_{\mathrm{R}}\right)\right)^{4}$.

The results of a scan through parameter space as $\Omega^{*}$ is varied may be summarised in a regime diagram with respect to $R o_{\mathrm{T}}$ and $\mathcal{T}_{\mathrm{F}}$, which identifies and classifies different circulation regimes according to their location in parameter space.

Figure 9 presents the regime diagram for the PUMA simulations of Wang et al. (2018), together with some sample snapshots of typical flows at different planetary rotation rates which present visualisations of the zonal wind speed at the upper tropospheric level of $200 \mathrm{hPa}$. As discussed more fully by Wang et al. (2018), the sequence of circulation patterns follows a clear trend with the planetary rotation rate. Keeping $\Delta \theta_{\mathrm{EP}}$ constant, the locus of varying rotation rate follows a diagonal path from the upper left to bottom right in $\left(\log R o_{\mathrm{T}}, \log \mathcal{T}_{\mathrm{F}}\right)$. At the lowest values of $\Omega^{*}$ the circulation is dominated by unsteady circumpolar vortices, a large-scale meridional overturning and strongly super-rotating zonal winds, both globally and locally (cf. Read and Lebonnois, 2018). These evolve into a pattern of more Earth-like, meandering mid-latitude jet streams at intermediate values of $\Omega^{*}$. This then breaks down into a more irregular pattern of parallel zonal jets, reminiscent of Jupiter and Saturn (although the latter both exhibit locally super-rotating equatorial jets, unlike the present model simulations), the number of which increases with increasing $\Omega^{*}$ values.

From an examination of the temperature and PV (potential vorticity) structure of the flow, it is evident that the basic circulation in these models allow for either barotropic or baroclinic instabilities, through satisfying either CSP criteria (i) or (iii). In the latter case, the method of diabatic forcing maintains a strong equatorward thermal gradient at the lower boundary which, for quasi-geostrophic conditions, enables
Table 1. Key dimensionless parameters for the baseline set of numerical simulations with $\Delta \theta_{\mathrm{EP}}=60 \mathrm{~K}, \tau_{\mathrm{ft}}=5$ Earth days and $\tau_{\mathrm{R}}=25.9$ Earth days, as defined by Eqs. (13) and (15).

\begin{tabular}{lrrr}
\hline$\Omega^{*}$ & $R o_{\mathrm{T}}$ & $\mathcal{T}_{\mathrm{F}}$ & $\mathcal{T}_{\mathrm{R}}$ \\
\hline $1 / 16$ & 20.5 & 238 & $1.71 \times 10^{5}$ \\
$1 / 8$ & 5.14 & $3.80 \times 10^{3}$ & $2.73 \times 10^{6}$ \\
$1 / 4$ & 1.28 & $6.09 \times 10^{4}$ & $4.67 \times 10^{7}$ \\
$1 / 2$ & 0.32 & $9.74 \times 10^{5}$ & $6.98 \times 10^{8}$ \\
1 & 0.08 & $1.56 \times 10^{7}$ & $1.12 \times 10^{10}$ \\
2 & 0.02 & $2.49 \times 10^{8}$ & $1.79 \times 10^{11}$ \\
4 & 0.005 & $3.99 \times 10^{9}$ & $2.86 \times 10^{12}$ \\
8 & 0.0013 & $6.38 \times 10^{10}$ & $4.58 \times 10^{13}$ \\
\hline
\end{tabular}

CSP criterion (iii) to be satisfied. Where a strong mid-latitude or circumpolar jet is formed, however, criterion (i) may be satisfied through lateral variation in the vorticity of the jet. Precisely which will dominate in particular cases, however, is not immediately clear without a more detailed analysis.

\subsection{Energetics}

Figure 10 presents the results of an analysis of the timeaveraged Lorenz energy cycles for each of a set of PUMA simulations from the work of Read et al. (2018) with fixed thermal forcing, covering a range of $\Omega^{*}$ from $1 / 16$ to 8 (representing a range in $R o_{\mathrm{T}}$ from $\sim 20-10^{-3}$; see Table 1). The energy content of the main reservoirs (Fig. 10a) is seen to change from a mainly KZ-dominated regime at low $\Omega^{*}$ values to a strongly AZ-dominated regime at fast $\Omega^{*}$ values $\left(R o_{\mathrm{T}} \ll 1\right)$. Both the eddy components $\mathrm{AE}$ and $\mathrm{KE}$ remain relatively small but rise to a peak for rotation rates corresponding to $R o_{\mathrm{T}} \sim 0.1-1$. All terms except AZ then decay strongly with increasing $\Omega^{*}$ values at the highest rotation rates.

Energy conversion rates exhibit even more complex variation with $\Omega^{*}$ (see Fig. 10b). For $R o_{\mathrm{T}} \gg 1$ the $\mathrm{CZ}$ term is strongly dominant, indicating a circulation that is energetically dominated by a thermally direct, zonal-mean meridional overturning. Within this range, eddies gain energy from the zonal-mean components through both the barotropic CK and baroclinic CE terms, but CK is dominant over CE (and CA) for $R o_{\mathrm{T}}>10$. At intermediate $\Omega^{*}$ values, the baroclinic conversions, $\mathrm{CE}$ and $\mathrm{CA}$, rise to a peak around $R o_{\mathrm{T}} \sim 0.3$, while $\mathrm{CK}$ and $\mathrm{CZ}$ decrease and actually change sign for $R o_{\mathrm{T}}<1$. This indicates that the energetics of the circulation are dominated by barotropically unstable eddies at the lowest rotation rates, although the baroclinic conversion term $\mathrm{CE}$ is also positive in this range, indicating a mixed barotropic/baroclinic instability as the origin of these eddies. For $R o_{\mathrm{T}} \lesssim 1$, however, CK becomes negative, while CE rises to a positive maximum around $R o_{\mathrm{T}} \sim 0.3$, indicating that eddies are predominantly baroclinic in character and energetics at relatively fast rotation rates. At the highest rotation rates 


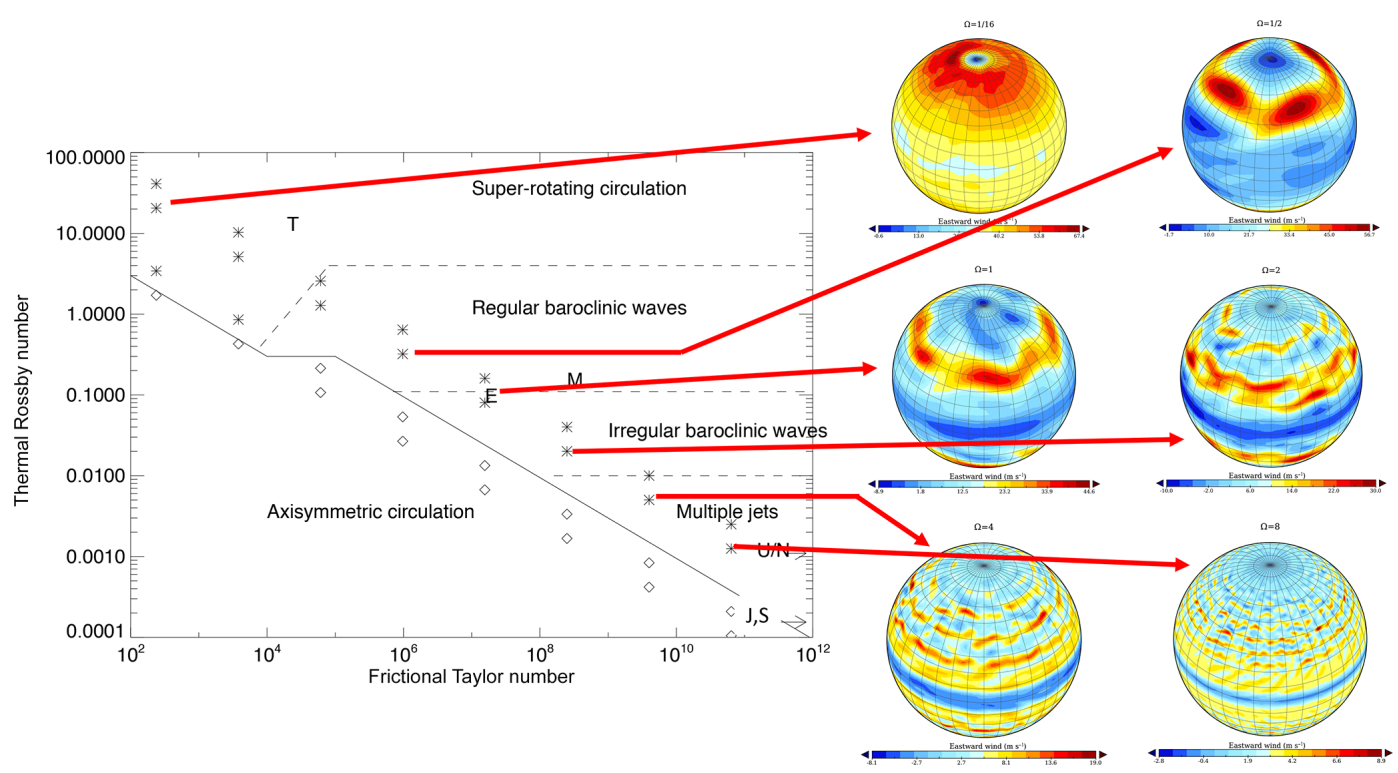

Figure 9. Regime diagram showing the various circulation regimes obtained in a simplified atmospheric general circulation model (Wang et al., 2018) with respect to characteristic dimensionless parameters $\left(R o_{\mathrm{T}}\right.$ and $\left.\mathcal{T}_{\mathrm{F}}\right)$. Stars refer to experiments in which wavy flows are observed, whereas open diamonds indicate experiments in which axisymmetric flows were found. The approximate locations in parameter space of some solar-system planets (Earth, Mars, Titan, Jupiter, Saturn, Uranus and Neptune) are labelled by their initial letters. The solid line delineates the boundary between axisymmetric circulations and circulations with wavy/turbulent flows. The dashed lines indicate the boundaries between different circulation regimes within the wavy/turbulent region. Some example snapshots of typical circulation patterns, visualised by shading of the zonal wind speed at the $200 \mathrm{hPa}$ level, are indicated by the arrows.

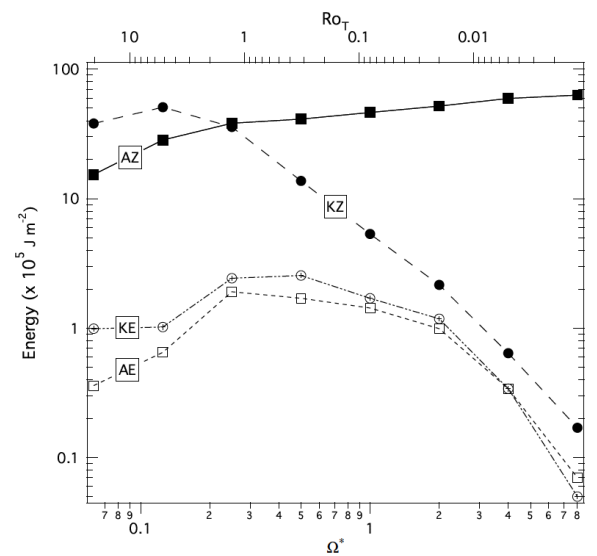

(a)

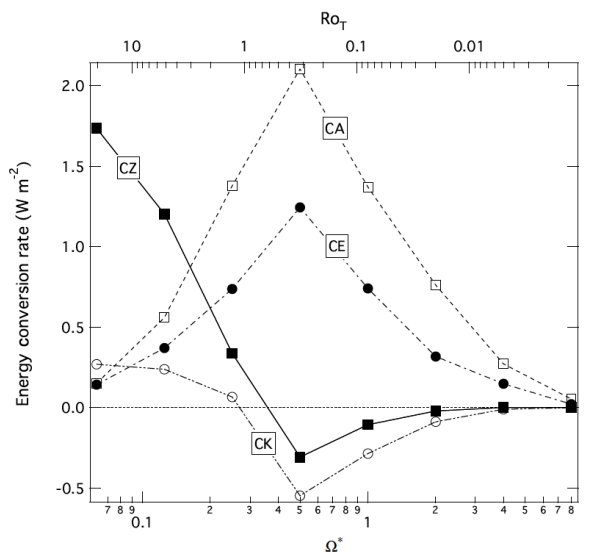

(b)

Figure 10. Terms in the Lorenz energy budgets for the series of PUMA simulations as a function of $\Omega^{*}$ and the thermal Rossby number: (a) globally averaged energies (in $10^{5} \mathrm{~J} \mathrm{~m}^{-2}$ ) and (b) the main energy conversion rates $\mathrm{CZ}, \mathrm{CA}, \mathrm{CE}$ and $\mathrm{CK}$. Conversion rates are in the unit of watts per square metre.

all conversion terms are seen to decrease in magnitude as $R o_{\mathrm{T}}$ decreases, leading to relatively weak and increasingly inefficient circulation.

\subsection{Heat transfer and baroclinic adjustment}

The positive contributions to both CA and CE at all rotation rates imply that the simulated circulation involves significant transfers of sensible heat across the planet. Figure 11a shows how the peak values of vertically integrated meridional heat transport vary with $\Omega^{*}$ and $R o_{\mathrm{T}}$ in the PUMA simulations of Wang et al. (2018). The zonal-mean and eddy heat transports are shown separately by open triangles and crosses respectively, with the total heat transport (zonal mean plus eddy) indicated by solid squares. This is similar in form to the results shown in Fig. 8a of Kaspi and Showman (2015) for their simulations using a GCM driven by a grey radiation scheme 


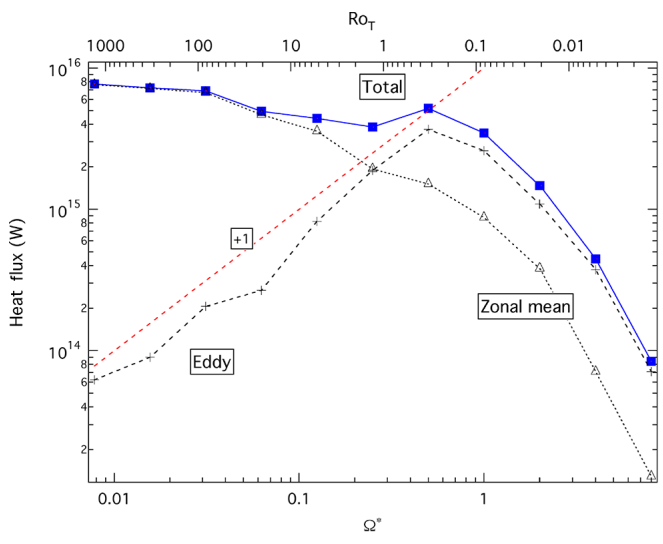

(a)

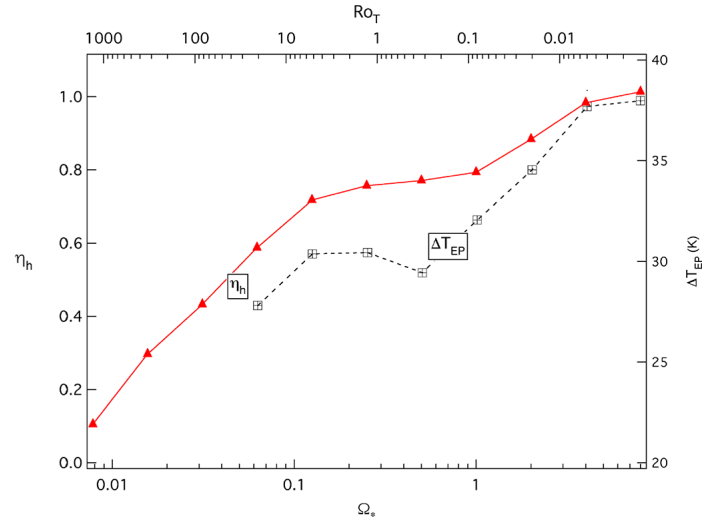

(b)

Figure 11. (a) Variations of peak values of meridional heat transport (W) within the PUMA GCM simulations of Wang et al. (2018) and at slower values of $\Omega$, shown as total poleward transport (blue squares), zonal-mean transport $\left(\int\left[c_{\mathrm{p}} \bar{v} \bar{T}\right] 2 \pi a \cos \left(\phi_{\max }\right) \mathrm{d} p / g\right)$ and eddy transport $\left(\int\left[c_{\mathrm{p}} \overline{v^{*} T^{*}}\right] 2 \pi a \cos \left(\phi_{\max }\right) \mathrm{d} p / g\right)$; (b) ratio $\eta$ of the slope obtained in fully active circulations in the latitude-height plane for experiments with different $\Omega^{*}$ values to the globally mass-weighted average isentropic slope in radiative-convective equilibrium (towards which the model atmosphere relaxes; shown as filled triangles connected by solid lines), plotted alongside the variation of mean equator-pole thermal contrast in the mid-troposphere, $\Delta T_{\mathrm{EP}}$ (shown as open squares connected by a dotted line). The (red) dashed line in (a) indicates a gradient of +1 with respect to $\Omega$, suggesting linear proportionality.

with moisture transport, apart from showing the combined total heat transport.

These results clearly show that, as in the simulations of Kaspi and Showman (2015), the zonal-mean contribution to heat transport dominates at slow rotation rates (for $R o_{\mathrm{T}}>$ 1 ) but decreases monotonically with increasing $\Omega^{*}$ values. The eddy contribution to heat transport, on the other hand, steadily increases (almost linearly) with $\Omega^{*}$ until it peaks around $R o_{\mathrm{T}} \sim 0.3$, beyond which it also decreases rapidly with increasing $\Omega^{*}$. The sum of the two contributions, however, remains nearly independent of $\Omega^{*}$ and $R o_{\mathrm{T}}$ until $R o_{\mathrm{T}} \sim$ 0.07 , indicating that for values of $R_{\mathrm{T}}$ between $\sim 1$ and 0.07 the eddy heat transport is able to compensate for the decrease in zonal-mean transport to maintain the total heat transport close to its slowly rotating value. At faster rotation rates, however, for which $R_{0} \lesssim 0.07$, eddies can no longer maintain this strength of heat transport, so the overall heat transport begins to drop.

Figure $11 \mathrm{~b}$ indicates that the global temperature structure also reflects these variations in total heat transport as $\Omega^{*}$ is varied. This shows $\eta$, a measure of the mean isentropic slope in the mid-troposphere $[\partial z / \partial y]_{\theta}$, normalised by the mean slope of the isentropes in the radiative equilibrium state to which the thermal state of the flow is relaxed and the mean equator-pole temperature contrast $\Delta T_{\mathrm{EP}}$ also in the mid-troposphere. These clearly show the isentropic slope and $\Delta T_{\mathrm{EP}}$ increasing with $\Omega^{*}$ but with a plateau in this variation for $R o_{\mathrm{T}}$ between $\sim 1$ and $\sim 0.1-0.05$. For $R o_{\mathrm{T}} \lesssim 0.05$, however, the reduction in heat transport as the baroclinically adjusted state breaks down leads to a resumption of the increase in isentropic slope with the rotation rate as the thermal struc- ture approaches the radiative-convective equilibrium configuration.

These results show a close similarity with the heat transport results discussed in Sect. 2.2 for thermally driven rotating annulus experiments and clearly indicate a regime in which eddy heat fluxes exert a strong influence on the thermal structure of the global circulation. This is despite an absence of a clear instability threshold for baroclinic instability in the GCM simulations, unlike what is found in the annulus experiments. However, the onset of this compensation effect of eddy heat fluxes against the decreasing zonal-mean transport (effectively acting as an "eddy thermostat") around a value of $R o_{\mathrm{T}} \simeq 1$ does correspond to where the vertical scale of Charney-type baroclinic instabilities first becomes of the same order as the pressure scale height and altitude of the tropopause (e.g. Branscombe, 1983) as $\Omega^{*}$ increases from smaller values. This would suggest that the baroclinic component of the eddies needs to span a large fraction of the active troposphere in order to be able to exert a strong influence on its thermal structure. But it does, therefore, indicate that when this condition is satisfied, it can exhibit a similar form of baroclinic adjustment to that found in the laboratory.

It would clearly be of interest to explore this trait in more detail in other, more realistic, GCM studies that also include the effects of radiative transfer and latent-heat transport.

\section{Baroclinic and barotropic instabilities in the solar system}

As discussed above in Sect. 1, Earth's atmosphere satisfies the CSP necessary conditions for instability mainly through 
criterion (iii), associated with persistent equatorward temperature gradients at the surface and the planetary vorticity gradient in the free atmosphere. The flanks of the upper-level mid-latitude jet might also lead to local changes of sign of $\partial Q / \partial y$ in the interior, while horizontal thermal gradients at the tropopause might also allow the CSP conditions to be satisfied at times through criterion (iv) (e.g. see Vallis, 2017, Sect. 9.9). These factors suggest multiple ways in which large-scale instabilities might occur in Earth's atmosphere, so which mechanism (baroclinic or barotropic) is likely to dominate?

Sections 2.2 and 3 both highlight the importance of the dimensionless parameters $B u$ and $R o_{\mathrm{T}}$ in favouring which mechanism is likely to be most important, although the distribution of $\partial Q / \partial y$ and its sign changes in the horizontal or vertical dimension also plays a role. Table 2 presents some rough estimates of the values of $B u$ and $R o_{\mathrm{T}}$ for Earth, Mars, Venus and Jupiter, based on Eqs. (13)-(15). These parameters for Earth are typically much less than $\mathrm{O}(1)$, which would tend to suggest that baroclinic instabilities dominate, as is well known (e.g. James, 1994; Vallis, 2017).

This is confirmed in typical calculations of the Lorenz energy budget for Earth's atmosphere. Figure 12a shows the result of a typical Lorenz energy cycle, as computed by Boer and Lambert (2008) from US National Centers for Environmental Prediction (NCEP) data, with energy reservoirs shown in units of joules per square metre and conversion rates in watts per square metre. This clearly shows that much of the convertible energy in the atmosphere resides in the AZ reservoir, which hosts more than twice as much as the rest of the reservoirs put together. The strongest internal conversions are the CA and CE terms, which indicate a net transfer of energy from zonal potential energy $\mathrm{AZ}$ to $\mathrm{KE}$ via $\mathrm{AE}$, which is the classical route representative of baroclinic instability (cf. Fig. 1d). The kinetic energy conversion CK is relatively small but robustly negative, indicating a transfer from KE to $\mathrm{KZ}$ consistent with the driving of a zonal-mean eddy-driven jet stream at mid-latitudes (e.g. James, 1994; Vallis, 2017). The AZ reservoir is maintained through the GZ term, representing the effects of differential radiative heating and cooling, while energy is ultimately removed from the system via the dissipative flux terms FZ and FE.

This pattern of energy reservoirs and fluxes is well known for Earth and is broadly consistent with the corresponding Earth-like case discussed in Sect. 3. But how is it for other planets in the solar system with substantial atmospheres which may be in very different circulation regimes from Earth? Where might they fit in comparison to the scenarios indicated by the regimes found with simplified GCMs, such as in Sect. 3?

\subsection{Mars}

Mars is arguably the most Earth-like planet elsewhere in the solar system, at least so far as its atmosphere and climate are concerned. It is roughly half the linear size of Earth (radius of $a \simeq 3400 \mathrm{~km}$ ) with a rotation period of just over $24 \mathrm{~h}$. It lies at a distance of around 1.3-1.5 astronomical units (AU) with an orbital period of around $687 \mathrm{~d}$, and with a rotation axis tilted by approximately $25^{\circ}$ from the perpendicular to its orbit, it experiences a strong seasonal cycle much like Earth. It possesses an atmosphere mainly consisting of $\mathrm{CO}_{2}$ with a surface pressure of around $6 \mathrm{hPa}$ which, although much less than on Earth, is sufficient to interact strongly with the rocky surface. Both water and $\mathrm{CO}_{2}$ can condense as ices to form clouds and surface deposits.

Its temperature structure and rapid planetary rotation lead to relatively small values of $R o_{\mathrm{T}}$ and $B u$ (see Table 2), though not as small as for Earth. Its relatively short radiative time constant leads to a value of $\mathcal{T}_{\mathrm{R}}$ which is much larger than $\mathrm{O}(1)$, indicating relatively weak radiative damping compared with Coriolis forces.

\subsubsection{PV structure}

Its similarities with Earth are also reflected in its potential vorticity structure, as can be seen e.g. in Fig. 13, which shows the zonal-mean, zonal wind and QGPV gradient for a typical atmospheric state during winter in the northern hemisphere on Mars, from data obtained from the Mars Climate Database (e.g. Lewis et al., 1999; see also http://www-mars. lmd.jussieu.fr, last access: 30 March 2020). As can be seen in Fig. 13a, zonal winds are typically concentrated into deep, mid-latitude jet streams during winter and the adjacent seasons, with strong vertical and horizontal shears and a pronounced latitudinal tilt with altitude. The corresponding structure in QGPV (see Fig. 13b) shows $\partial Q / \partial y(\partial Q / \partial \phi)$ positive in the jet itself (cf. Fig. 13a) but with weaker reversals in sign on either side. Together with the positive equatorward temperature gradient at the surface in the north, this would indicate that the CSP conditions can be satisfied in this season either by criterion (i) or (iii), much as on Earth. The atmosphere might then be expected to exhibit either baroclinic or barotropic instabilities, or a mixture of both, although the linear instability analysis of Barnes (1984) suggests that the baroclinic instability mechanism dominates for Martian conditions, while the barotropic shear mechanism acts to damp the baroclinic instability.

It is interesting to note that, at this time during southern summer, the corresponding zonal jet stream becomes much weaker and even reverses direction in places. The QGPV gradient does exhibit a weak reversal in sign, though the equatorward thermal gradient near the surface also reverses in sign during summer so that conditions are much less favourable for baroclinic instability via CSP criterion (iii). This is consistent with the observed near suppression of baroclinic instability in Martian summers at mid-latitudes (e.g. Lewis et al., 2016), except for occasional very shallow disturbances that are sometimes observed on small horizontal scales close to the polar cap edge (e.g. Gierasch et al., 1979b). 
Table 2. Estimates of the main dimensionless parameters for Earth, Mars, Venus and Jupiter. Estimates of the radiative time constant are based on values from NASA's Planetary Data System (PDS; see https://pds-atmospheres.nmsu.edu/education_and_outreach/encyclopedia/ radiative_time_constant.htm, last access: 30 March 2020).

\begin{tabular}{lrrrr}
\hline Planet & $B u$ & $R o_{\mathrm{T}}$ & $\tau_{\mathrm{R}}(\mathrm{s})$ & $\mathcal{T}_{\mathrm{R}}$ \\
\hline Earth & 0.02 & 0.06 & $4.1 \times 10^{6}$ & $3 \times 10^{10}$ \\
Mars & 0.04 & 0.20 & $1.9 \times 10^{5}$ & $1.3 \times 10^{5}$ \\
Venus (planet) & 140 & 370 & $2.2 \times 10^{5}-4 \times 10^{9}$ & $7.5 \times 10^{-5}-8 \times 10^{12}$ \\
Venus (cloud tops) & 0.04 & 0.10 & $2.2 \times 10^{5}$ & $10^{3}$ \\
Jupiter (cloud tops) & $8 \times 10^{-4}$ & $10^{-3}$ & $1.4 \times 10^{8}$ & $1.5 \times 10^{18}$ \\
\hline
\end{tabular}

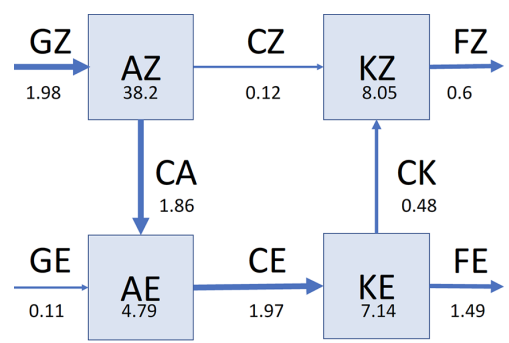

(a)

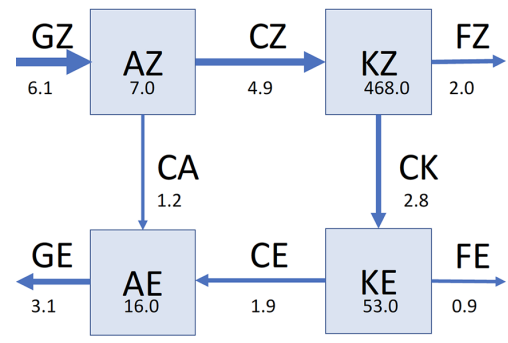

(c)

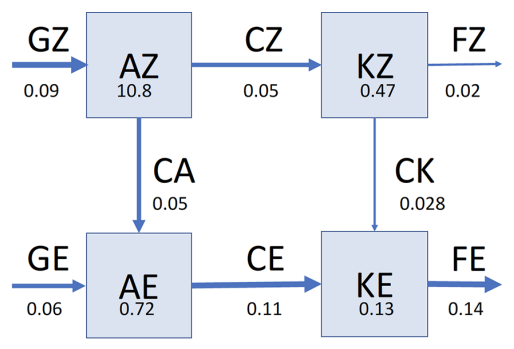

(b)

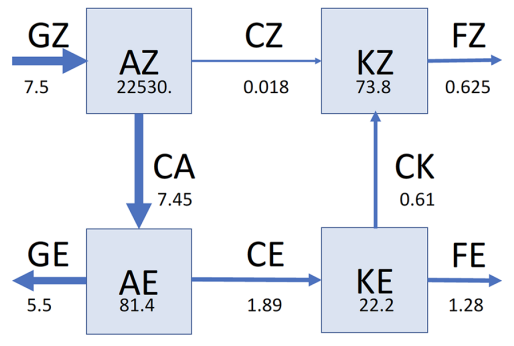

(d)

Figure 12. Representations of energy storage and interconversions in the form suggested by Lorenz (1955, 1967) for a selection of the main planets of the solar system: (a) Earth (calculations from Boer and Lambert, 2008); (b) Mars (calculations from Tabataba-Vakili et al., 2015); (c) Venus (calculations from Lee and Richardson, 2010) and (d) Jupiter's weather layer (from preliminary calculations using a higherresolution version of the model described in Young et al., 2019a). As before, energy reservoirs are shown in units of $10^{5}$ joules per square metre, and conversion rates and source/sink terms are in units of watts per square metre.

\subsubsection{Energy budget}

The corresponding Lorenz energy budget is needed to establish which is the dominant mechanism to energise the Martian eddy circulation. This has been computed recently by Tabataba-Vakili et al. (2015), based on an assimilated analysis of orbiting spacecraft measurements of Mars over a period of at least 3 Mars years (Montabone et al., 2014). The data were assimilated into the UK version of the Laboratoire de Météorologie Dynamique (LMD) Mars GCM to produce a daily record of Martian meteorology over the entire period, from which the energy budget could be computed (see Fig. 12b).

The results, illustrated e.g. in Fig. 12b, show a qualitatively similar pattern to that of Earth, with the majority of dynami- cally active energy in the atmosphere stored as zonal potential energy AZ. The dominant conversion terms are the CA and $\mathrm{CZ}$ conversions from $\mathrm{AZ}$ to $\mathrm{AE}$ and $\mathrm{KZ}$ respectively (at $50 \mathrm{~mW} \mathrm{~m}^{-2}$ ) and especially the CE term (at $110 \mathrm{~mW} \mathrm{~m}^{-2}$ ). This indicates a strong energetic role for the thermally direct axisymmetric (Hadley cell) overturning circulation as well as the principal baroclinic conversions CA and CE maintaining synoptic eddies. This confirms the role for baroclinic instability as suggested from the configuration of potential vorticity gradients in Fig. 13b. The barotropic eddy-zonal conversion $\mathrm{CK}$, however, is positive and around half the amplitude of CA, suggesting a role for exchanges consistent with a mixed baroclinic-barotropic instability. The GE source term for $\mathrm{AE}$ is also quite substantial and of the same order as $\mathrm{CA}$ and $\mathrm{CZ}$. This is because the day-night contrast in so- 


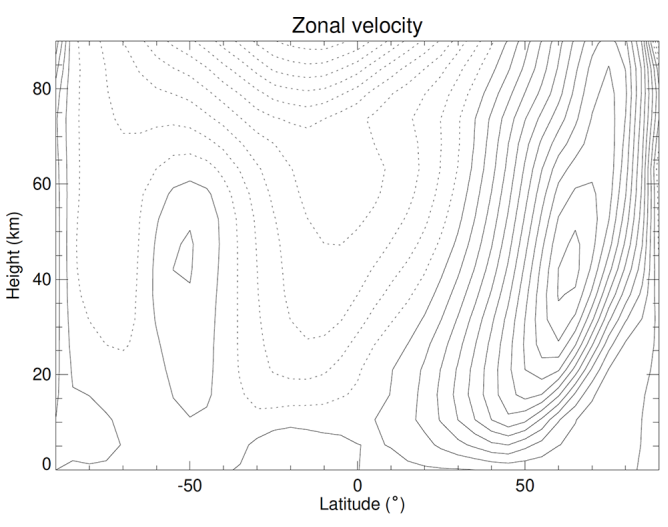

(a)

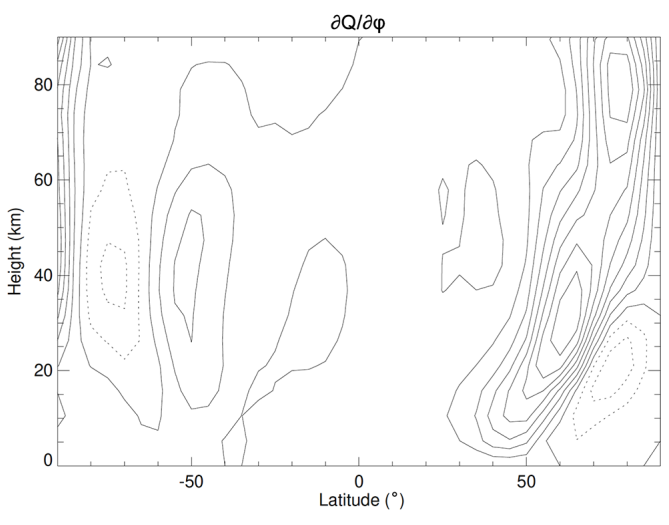

(b)

Figure 13. Zonal-mean sections of (a) zonal wind $\left(\mathrm{ms}^{-1}\right)$ and (b) $\partial \bar{Q} / \partial \phi\left(\mathrm{s}^{-1} \mathrm{rad}^{-1}\right)$ typical of winter in the Martian northern hemisphere. Data were taken from the Mars Climate Database (e.g. Lewis et al., 1999); see also http://www-mars.lmd.jussieu.fr/mars/access.html, last access: 30 March 2020 for $L_{\mathrm{s}}=270^{\circ}$ using the standard Mars climatology. Contour intervals are (a) $15 \mathrm{~m} \mathrm{~s}^{-1}$ and (b) $0.0002 \mathrm{~s}^{-1} \mathrm{rad}^{-1}$, and negative contours are shown dashed.

lar heating on Mars generates a strong thermal tide response which contributes to the AE and KE reservoirs, although this varies significantly with season and the amount of dust loading in the atmosphere. As discussed by Tabataba-Vakili et al. (2015), this budget does exhibit fairly significant seasonal variations, also between the northern and southern hemispheres, but the overall structure of the energy budget remains largely unchanged.

\subsection{Venus}

Venus is the other main terrestrial planet in the inner solar system that possesses a substantial atmosphere. It is also Earth-like in some respects but very different in others. Around the same size as Earth, Venus is somewhat closer to the Sun than Earth at a mean distance of $0.72 \mathrm{AU}$ and orbits the Sun in around $224.7 \mathrm{~d}$. The planetary rotation period, however, is very long compared to Earth, with a sidereal rotation period of $243.7 \mathrm{~d}$ in a retrograde sense and a very small obliquity angle $\left(177.3^{\circ}\right.$, equivalent to $2.6^{\circ}$ if it rotated in the same sense as its orbit).

Its atmosphere is deep and massive, consisting mainly of $\mathrm{CO}_{2}$ with a surface pressure of more than 90 bars $^{1}$ and typical surface temperature of $740 \mathrm{~K}$. The entire planet, however, is almost completely shrouded in thick clouds, thought to consist mainly of aqueous sulfuric acid droplets that scatter sunlight very efficiently (with a Bond albedo of 0.77 ), and located between 40 and $60 \mathrm{~km}$ above the surface. Dynamically, Venus is in a very different regime compared to Earth. Its winds are dominated by a very rapid super-rotation with maximum zonal winds of more than $100 \mathrm{~m} \mathrm{~s}^{-1}$, located around or slightly above the main cloud decks at altitudes

\footnotetext{
${ }^{1}$ The bar is a unit of pressure approximately equal to the mean atmospheric pressure at Earth's surface and is equivalent to $1000 \mathrm{hPa}$.
}

of $50-70 \mathrm{~km}$. This means that the atmosphere at the cloud level rotates around 60 times faster than the underlying surface. The processes driving such a remarkable circulation are still imperfectly understood, though likely entail the role of waves and eddies on various scales (e.g. see Sánchez-Lavega et al., 2017; Read and Lebonnois, 2018, for recent reviews). Despite such strong winds and dynamical activity, horizontal temperature gradients are relatively weak compared with those in the vertical direction, with typical equator-to-pole thermal contrasts of no more than 10-20 K.

These factors lead to somewhat different estimates of some of the key dynamical parameters, depending upon whether the atmosphere is viewed in the frame of the underlying planet or in corotation with the main cloud deck. In the frame of the planet, the values of $B u$ and $R o_{\mathrm{T}}$ are likely to be much larger than $\mathrm{O}(1)$ (although $N$ is also close to zero in the deep troposphere), indicating that the flow is unlikely to be geostrophic (its cloud level zonal winds are predominantly in cyclostrophic balance with centrifugal accelerations dominating over Coriolis accelerations; e.g. see Sánchez-Lavega et al., 2017) or baroclinically unstable, although it could be consistent with various forms of barotropic shear instability. If the circulation is viewed in the average frame of the cloud level winds (e.g. see Young et al., 1984), however, which rotate around the planet on average in around $4 \mathrm{~d}$ (corresponding to $f=2 \Omega \simeq 3.6 \times 10^{-5} \mathrm{~s}^{-1}$ ), a different perspective emerges with typical values of $B u$ and $R o_{\mathrm{T}}$ of around 0.04-0.1. In this frame, therefore, the cloud level circulation may appear to be in quasi-geostrophic balance with the possibility of baroclinic instabilities that would likely be localised in the vertical direction around the levels of the main cloud decks, provided the potential vorticity configuration would allow the CSP necessary conditions for instability to be satisfied. 


\subsubsection{PV structure}

A key difficulty in determining the PV structure of Venus's atmosphere arises from a lack of detailed observations of winds and temperature within and beneath its main cloud decks (e.g. see Sánchez-Lavega et al., 2017). It is necessary, therefore, to fall back on numerical models of Venus's atmospheric circulation for the kind of information needed to compute quantities such as $\partial Q / \partial y$. But the numerical simulation of Venus's atmospheric circulation is still at a relatively immature state, with realistically forced models appearing only recently (e.g. Lebonnois et al., 2010, 2016; Mendonça and Read, 2016). Simpler models have been studied more extensively but have struggled to reproduce key features of the circulation and have exhibited significant divergence among different model formulations (e.g. Lee and Richardson, 2010; Lebonnois et al., 2013; Lewis et al., 2013). This may reflect important differences e.g. in the computation of static stability (and hence the ratio $f / N$ ) and in how well different dynamical cores are able to conserve and transport angular momentum.

For the present purpose, therefore, we follow Young et al. (1984) and Sugimoto et al. (2014) in examining a somewhat idealised form of the zonal-mean state of a Venus-like atmosphere. This is illustrated in Fig. 14, taken from the work of Sugimoto et al. (2014), and shows a zonal-mean section of the zonal wind (Fig. 14a) and corresponding field of $\partial Q / \partial \phi$ (in the average frame of the zonal wind at an altitude of $56 \mathrm{~km}$; Fig. 14b). The zonal wind section (in the planetary reference frame) captures the main features of the observed zonal winds on Venus, with a monotonic growth of zonal wind strength with altitude at all latitudes towards maximum values of $100-120 \mathrm{~m} \mathrm{~s}^{-1}$ at around $70 \mathrm{~km}$ altitude but with the formation of jet-like features at mid-latitudes in both hemispheres. This idealisation then neglects any vertical shear above the cloud tops, although this may not be particularly realistic. However, the flow structure around the cloud top altitudes are of most interest here. The winds are constructed to be in gradient wind balance (between horizontal pressure gradients and a combination of Coriolis and centrifugal "forces") with the corresponding pressure and temperature fields.

The distribution of $\partial Q / \partial \phi$ in Fig. 14b indicates a concentration of the PV gradient at high latitudes, especially within the cloudy layers between altitudes of 55 and $65 \mathrm{~km}$ (although the clouds are not represented explicitly in this model). Moreover, this gradient clearly changes sign in the vertical direction while remaining positive over most of the domain at lower levels. This would immediately suggest the possibility of a baroclinic instability localised within the cloud deck levels by satisfying the CSP conditions for instability via criterion (i). A change of sign in the horizontal is only discernible in the layer around $56 \mathrm{~km}$, however, suggesting that barotropic instabilities might also be possible, though again centred mainly within the cloud decks. Nearer the surface, $\partial Q / \partial \phi>0$, but surface temperature gradients are very weak, suggesting that Venus may be able to satisfy the CSP condition via criterion (iv) only marginally. Besides, the flow is far from being in geostrophic balance at these levels, although that would not necessarily rule out the possibility of a non-geostrophic form of baroclinic instability.

\subsubsection{Energy budget}

The energy budget for Venus is similarly not available from observations, and so we must rely on model simulations to compute the likely energies and exchanges. To the authors' knowledge, this has not so far been done for any realistically forced, comprehensive GCM of Venus that faithfully reproduces the observed winds and circulation. However, Lee and Richardson (2010) did compute Lorenz energy budgets for a set of somewhat simplified GCM simulations of Venus-like circulations, and so this is what is presented here. However, these model simulations did not fully represent the radiative forcing of the atmosphere, including its diurnal cycle, nor did they fully capture the observed distribution of angular momentum in the simulations. So the budget shown here is likely to be inaccurate in some degree and missing some key processes. Nevertheless, the results are worthy of attention as a first step towards a more accurate calculation.

Figure 12c presents the energy budget calculations for the spectral core case of Lee and Richardson (2010) with full damping as being reasonably representative of this class of Venus model (without an explicit diurnal cycle). The energy reservoirs show a very different distribution of energy from either Earth or Mars, with most of the dynamically active energy in the atmosphere residing in the zonal and eddy kinetic energies $\mathrm{KZ}$ and KE. The potential energy reservoirs, however, are certainly not negligible and suggest a significant concentration of available potential energy in the AE reservoir. AZ is relatively small, though, as would be expected for an atmosphere with relatively weak meridional temperature gradients. Among the conversion terms, CZ is the largest, indicative of a strong, thermally direct Hadley overturning circulation to maintain the very large $\mathrm{KZ}$ reservoir. The next largest conversion is $\mathrm{CK}$, which evidently acts in the positive direction to convert from $\mathrm{KZ}$ to $\mathrm{KE}$. This would suggest a dominance of barotropic processes, much as found in the idealised Earth-like simulations of Sect. 3 at low values of $\Omega^{*}$.

$\mathrm{CA}$, on the other hand, also acts in a positive direction (from $\mathrm{AZ}$ to $\mathrm{AE}$ ), indicating some alignment with baroclinically active processes and perhaps suggesting a mixed baroclinic/barotropic character to the dominant eddy processes. $\mathrm{CE}$, however, acts in a negative direction, from $\mathrm{KE}$ to $\mathrm{AE}$, which would suggest that mechanical exchanges between eddy potential and kinetic energies dominate over buoyancy-driven instabilities. The one term that may be anomalous when compared with Venus is the potential energy source/sink GE. This appears to be acting to remove 


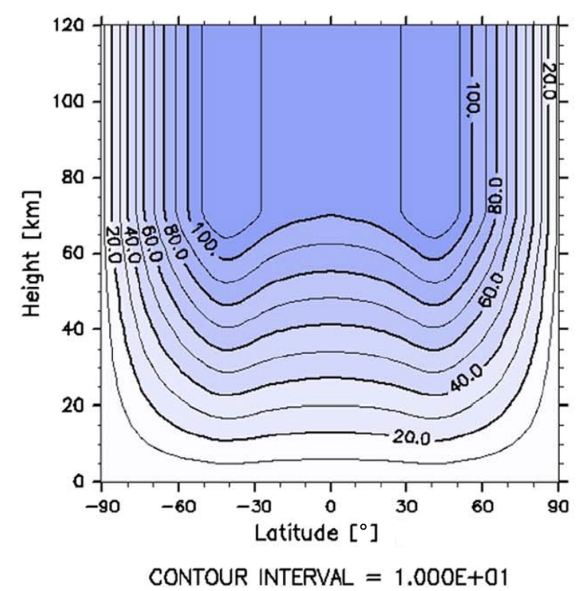

(a)

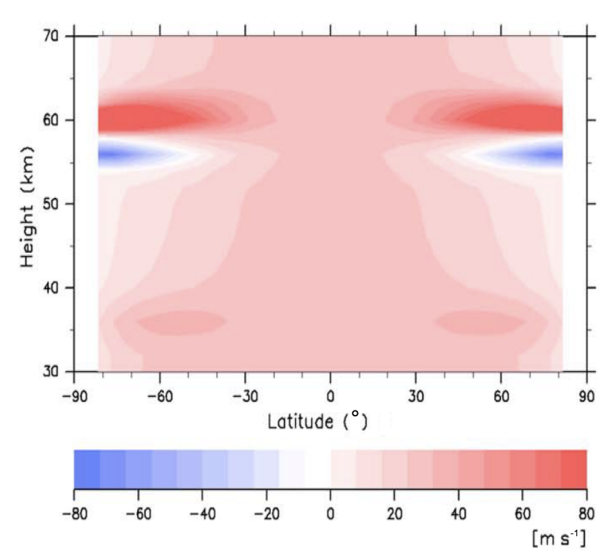

(b)

Figure 14. Zonal-mean sections of (a) zonal wind $\left(\mathrm{m} \mathrm{s}^{-1}\right)$ and (b) $a \partial Q / \partial \phi\left(\mathrm{m} \mathrm{s}^{-1}\right)$ typical of Venus's global circulation, as presented by Sugimoto et al. (2014) from their numerical model simulations.

energy from $\mathrm{AE}$ at a fairly strong rate. But this model takes no account of the diurnal cycle and hence the input of thermal energy to the thermal tides. More realistic model simulations suggest that the thermal tides in and above the main cloud decks play an important role in maintaining the observed very strong atmospheric super-rotation (e.g. SánchezLavega et al., 2017). It seems more likely, therefore, that a more complete simulation of the Venus circulation would include a large positive contribution to GE, much as indicated for Mars in Fig. 12b. This is a question that should be followed up urgently through analysis of more realistic model simulations.

\subsection{Jupiter}

Jupiter is the largest of a quite different class of planet within the solar system, known as the gas giants, which also includes Saturn but also shares a number of dynamical aspects in common with Uranus and Neptune, known as the ice giants because of differences in composition and internal structure from Jupiter and Saturn (e.g. Guillot, 2005). As these names would suggest, these planets are much larger than Earth (Jupiter's mass is around 316 times larger than Earth's), with the gas giants largely composed of hydrogen and helium in proportions similar to the Sun. Both gas and ice giants therefore do not support a solid surface, unlike the terrestrial planets, but remain fluid to great depths until reaching a core of heavier elements close to the planetary centre. At a certain depth, however, the highly compressed fluid becomes electrically conducting, and at even greater depths the hydrogen undergoes a phase change from a supercritical molecular fluid to a liquid metal (at least for Jupiter and Saturn, though not for the so-called ice giants, Uranus and Neptune; e.g. see Guillot, 2005). All four gas and ice giant planets orbit far from the Sun, with Jupiter (the innermost of these plan- ets) in an orbit of mean radius 5.2 AU and orbital period of 11.86 years.

All four gas and ice giant planets are fully cloud covered, with clouds composed mainly of chemically reduced compounds such as ammonia ice, $\mathrm{NH}_{4} \mathrm{SH}, \mathrm{H}_{2} \mathrm{~S}$ and water (and $\mathrm{CH}_{4}$ on Uranus and Neptune). Motions of these clouds reveal a very different circulation at the tops of their tropospheres from what is seen on Earth or the other terrestrial planets. Winds are predominantly zonal and arranged in systems of alternating eastward and westward jet streams, on scales smaller than the planetary radius. Cloud bands partly align with these zonal flows but are perturbed by waves and oval vortical eddies across a range of scales. Near the main cloud decks, incoming sunlight contributes significantly to the radiative energy budget. However, both Jupiter and Saturn (and Neptune) are significant net emitters of excess thermal radiation from a heat source in the deep interior, most likely representing residual thermal energy still escaping to space from the time of the original formation of these planets (e.g. Guillot, 2005). The action of incoming sunlight, upwelling heat from the deep interior and even the release of latent-heat energy from the condensation of trace amounts of water in these planets have been suggested to play an important role in an atmospheric layer around $200-300 \mathrm{~km}$ thick, commonly known as the "weather layer" (e.g. Gierasch et al., 2000; Ingersoll et al., 2000). In the latter case, deep convective clouds and lightning are observed on Jupiter and Saturn which some estimates (Gierasch et al., 2000) suggest might carry as much as half of the upward thermal energy flux from the deep interior into the weather layer. The extent to which such convective storms energise large-scale motions on Jupiter or Saturn remains controversial, however, since the apparent horizontal scale of such moist convective clusters is likely significantly smaller than the $\sim 3000 \mathrm{~km}$ scale inferred by Young and Read (2017) for the excitation of Jupiter's in- 
verse kinetic energy cascade. The zonally dominated circulation in this weather layer is not thought to penetrate very deeply below the bottom of this layer, though recent measurements from the Juno mission suggest that the zonal wind pattern could penetrate as much as $3000 \mathrm{~km}$ below the visible clouds (around $4 \%$ of Jupiter's radius; Kaspi et al., 2018), though this may be still somewhat uncertain (e.g. see Kong et al., 2018).

Despite their massive sizes, both Jupiter and Saturn are very rapid rotators with rotation periods of around $10 \mathrm{~h}$. This would imply that differential motions within their fluid envelopes are highly likely to be strongly geostrophic. The observable regions of their atmospheres, above, within, and somewhat below the main $\mathrm{NH}_{3}$ and $\mathrm{H}_{2} \mathrm{O}$ cloud decks are found to be stably stratified, indicating the possibility of instability processes that have dynamical properties in common with those found in Earth's atmosphere and oceans. Their rapid rotation leads to values of the main Rossby radius of deformation $L_{\mathrm{D}}$ to be much smaller than the planetary radius $a$. This is reflected in the very small values of $B u$ and $R o_{\mathrm{T}}$ indicated for Jupiter's cloud tops in Table 2. Together with the very large value of $\mathcal{T}_{\mathrm{R}}$, this would seem to suggest that geostrophic forms of barotropic, and possibly baroclinic, instabilities are likely to occur in the weather layers of these planets.

\subsubsection{PV structures: observations}

The occurrence of such instabilities, however, depends crucially on the distribution of PV within the weather layers of these planets. As with the other planets under discussion here, Jupiter's atmosphere needs to satisfy the CSP necessary condition for instability somehow. Without a solid surface, however, CSP criterion (iii) cannot be satisfied, unlike on Earth or Mars. Such an argument was used by Gierasch et al. (1979a) to suggest only a minor role at most for baroclinic instabilities on Jupiter. But as subsequently noted by Conrath et al. (1981), the possibilities of satisfying the CSP condition through criteria (i) or (ii) remain, especially if Jupiter or Saturn possess a strong tropopause which can act as an internal interface that supports a horizontal thermal gradient. The analysis of Conrath et al. (1981) noted that, in the absence of a significant horizontal shear and with a lower boundary that was essentially isentropic and weakly stratified, westward jets could satisfy the CSP condition through criterion (i) (or (ii) if the tropopause is considered as an upper boundary or interface). Their calculations indicated that such an "inverted Charney" form of baroclinic instability could have relatively fast growth rates and even lead to up-gradient momentum fluxes for instabilities on a small enough horizontal scale.

The vorticity structure of the zonal-mean flow at the cloud tops has been measured since the time of the Voyager spacecraft encounters with Jupiter in 1979. By tracking features in the ubiquitous ammonia cloud decks in successive images taken by the spacecraft, the pattern of eastward and westward jet streams could be derived as a profile in latitude from which the vorticity and its northward gradient could be obtained (e.g. Ingersoll et al., 1981; Salyk et al., 2006; Galperin et al., 2014). These profiles indicate that the northward gradient of absolute vorticity robustly changes sign with latitude in a number of places, typically around the peaks of westward jets, leading to some suggestions that these jets may be prone to barotropic instabilities, since they would appear to satisfy criterion (i) for the CSP instability condition (Ingersoll et al., 1981). The stretching term associated with the thermal structure of the flow may also be important, leading to some suggestions (e.g. Ingersoll and Cuzzi, 1969; Scott and Dunkerton, 2017) that vigorous lateral mixing of PV could result in a monotonic, staircase-like variation of $Q$ with latitude which would be unconditionally stable to shear instabilities. Such a process that might hold the atmosphere close to a state of marginal instability would effectively constitute a form of barotropic adjustment by analogy with Stone's baroclinic adjustment hypothesis (Stone, 1978).

Observational studies that have combined wind measurements from cloud motions with remotely sensed temperature retrievals (Read et al., 2006, 2009a; Antuñano et al., 2019) and a geostrophic balance assumption for both Jupiter and Saturn, however, also indicate significant reversals of $\partial Q / \partial y$. Figure 15a shows an example of a section of $\partial Q / \partial y$ in latitude and height in the southern hemisphere of Jupiter, based on data from the Cassini spacecraft (Read et al., 2006). This clearly shows patches where $\partial Q / \partial y<0$ overlying westward jets but persisting over significant ranges in altitude. $\partial Q / \partial y$ even appears to change sign in the vertical direction in some locations in Jupiter's stratosphere, although these observations should be treated with caution, since the reconstruction of the flow at high altitudes requires the integration of the geostrophic thermal wind shear relation which can amplify the effects of measurement noise and uncertainties, especially in quantities that require spatial differentiation (e.g. see Read et al., 2009a). Being based on nadir observations, the retrieved thermal measurements also have rather limited vertical resolution. So it cannot be ruled out that structures associated with sharp thermal gradients along the lines suggested by Scott and Dunkerton (2017) might exist that would eliminate such reversals in the sign of $\partial Q / \partial y$.

The distribution of $Q$ beneath Jupiter's cloud tops, however, is virtually unknown because of a dearth of detailed measurements of winds, temperatures or composition. But there seems no reason a priori to conclude that internal changes of sign of $\partial Q / \partial y$ in the deep troposphere, or of $\partial T / \partial y$ at the tropopause, cannot exist and that therefore the CSP necessary condition for baroclinic or barotropic instability could be satisfied through criteria (i) or (ii). 

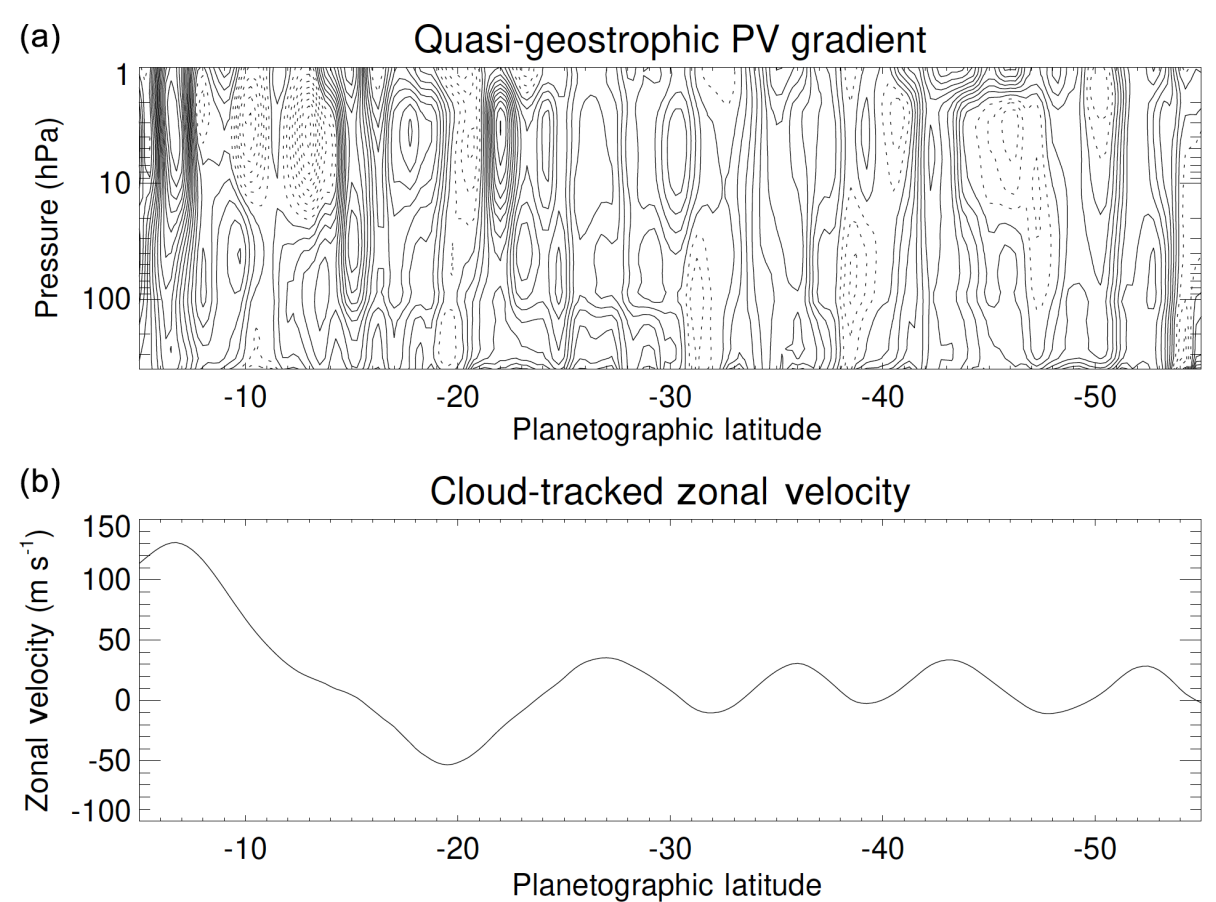

Figure 15. (a) Zonal-mean section of $\partial Q / \partial y\left(\mathrm{~s}^{-1} \mathrm{~m}^{-1}\right)$ and (b) zonal-mean zonal wind $\left(\mathrm{ms}^{-1}\right)$ in the southern hemisphere of Jupiter, derived from Cassini observations by Read et al. (2006).

\subsubsection{PV structures: GCMs}

A number of studies have appeared recently in which fully three-dimensional, time-dependent GCMs have been developed of Jupiter's or Saturn's weather layers which seek to capture the thermal and vorticity structures of the flow around and beneath the cloud tops (e.g. Liu and Schneider, 2010; Lian and Showman, 2008, 2010; Palotai et al., 2014; Young et al., 2019a, b; Spiga et al., 2020). These models have included the effects of solar heating in the stratosphere and upper troposphere and upwelling internal heating from the deep interior and have been capable of capturing a number of realistic features such as the equatorial prograde jets and multiple eddy-driven zonal jets at mid-latitudes.

Figure 16 shows a cross section of $\partial Q / \partial y$ and $\bar{u}$ from a GCM simulation of Jupiter's weather layer by Young et al. (2019a) (their run B, with a horizontal resolution of $512 \times 256$ points in longitude and latitude). As with the observations, this shows a generally clear correlation between $\bar{u}$ and $\partial Q / \partial y$ in the upper troposphere but with some sign reversals of $\partial Q / \partial y$ in both the horizontal and vertical in relation to both westward and eastward jets in several cases. This would seem to confirm that the simulated flow sustains the necessary conditions for baroclinic instability through CSP criterion (i) as discussed above.

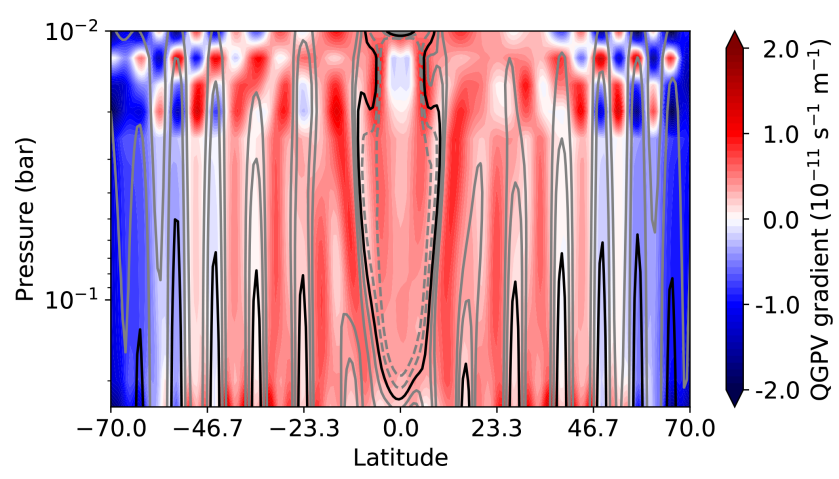

Figure 16. Zonal-mean latitude-height section of $\partial Q / \partial y$ $\left(\mathrm{s}^{-1} \mathrm{~m}^{-1}\right)$ and zonal-mean zonal wind $\left(\mathrm{m} \mathrm{s}^{-1}\right)$ in a numerical simulation of Jupiter's weather layer, from Young et al. (2019a). Data correspond to run B of Young et al. (2019a) and were averaged over the last $100 \mathrm{~d}$ of the equilibrated run. Shading represents $\partial Q / \partial y$, and the line contours represent zonal-mean zonal velocity $\bar{u}$, shown for $-10,-5,+5$ and $+10 \mathrm{~m} \mathrm{~s}^{-1}$ in grey and $0 \mathrm{~m} \mathrm{~s}^{-1}$ in black.

\subsubsection{Energy budget}

A preliminary Lorenz energy budget, computed using an updated, higher-resolution version of the Jupiter weather layer model described by Young et al. (2019a), is shown in Fig. 12d. This represents the model's attempt to capture the energetics of a Jupiter-like weather layer between the pressure levels of 18 bars and $10 \mathrm{hPa}$. The energy reservoirs reflect a distribution of potential and kinetic energy typical of 
a rapidly rotating, stratified atmosphere, with most of the stored energy residing in the zonal-mean potential energy AZ. Smaller amounts are found in the $\mathrm{KZ}$ and $\mathrm{AE}$ reservoirs with KE being the weakest, though still containing much more energy per unit area than the corresponding reservoirs for Earth.

Energy conversions, however, are remarkably Earth-like in their relative sizes and directions, with $\mathrm{CA}$ and $\mathrm{CE}$ as the largest conversion rates in the sense expected for baroclinic instabilities. This confirms the indication from the PV structure that anticipated that baroclinic instabilities could occur and play a significant role in the circulation. CK acts to maintain the zonal-mean flow through transfers from KE, at a vertically integrated rate $\left(0.61 \mathrm{~W} \mathrm{~m}^{-2}\right)$ that is not unreasonable with regard to the observed transfer rate near the cloud tops (e.g. Ingersoll et al., 1981; Salyk et al., 2006; Galperin et al., 2014). CZ acts in the thermally direct sense but is relatively weak, reflecting the comparatively minor role played by zonally symmetric overturning circulations in the global circulation. The largest terms overall are CA, GZ and GE, indicating some very strong forcing of the zonal-mean potential energy field and conversion to eddy potential energy, much of which is then extracted by diabatic processes. A complication is that this model simulation includes both latent-heat fluxes and exchanges associated with a moist convection parameterisation, which may not be fully accounted for in the conventional Lorenz energy budget approach. But the overall structure of this cycle strongly suggests an important role for baroclinic instability, at least in this model simulation of Jupiter's weather layer.

\section{Conclusions}

Throughout this review it has been clear that baroclinic and/or barotropic instabilities play a key role in generating some of the most energetic eddies and waves in most rotating, stratified systems, including systems that are very different from Earth's atmosphere and oceans. The distinction between baroclinic and barotropic instabilities is, in certain respects, an artificial one, at least concerning the constraints deriving from the vorticity structure of the flow. The principal distinction is a real one, however, based largely upon whether the dominant conversion of energy from an initial basic state is from its stored potential or kinetic energy, as revealed in their Lorenz energy budgets. But the CSP necessary condition for instability applies to both mechanisms, which are governed by similar vorticity constraints. In practice, eddy formation in realistic systems with both vertical and horizontal shear could entail contributions from both potential and kinetic energy stored in the basic state so that it is natural to consider either mechanism as different facets of a common process. This suggests the value of a unifying approach, although a number of outstanding issues remain.

\subsection{Stability theory}

The body of stability theory continues to provide important and useful guidance to help predict the onset of instabilities in various practical geophysical and astrophysical contexts and in particular to establish conditions for when instabilities will not occur. But there are still many situations that are not fully worked out in complete generality, and most published stability criteria have shortcomings that fail to take certain factors into account. After more than 50 years, the CSP instability condition is still widely used to assess the stability of zonal flows in atmospheres and oceans, even though it is based on linearised quasi-geostrophic theory for perturbations to a purely axisymmetric basic state. As an instability condition it only represents a necessary condition but not a sufficient one to guarantee instability if it is met.

The more sophisticated stability theorem of Arnol'd (1966) and more recent extensions (e.g. see $\mathrm{Mu}$ and $\mathrm{Wu}$, 2001, for a review) offer a more refined approach which takes some account of nonlinearity and non-zonal structure, although with some non-trivial restrictions. It is most well developed for quasi-geostrophic flows, but a full treatment, e.g. of the primitive equations of dynamical meteorology, is still lacking. Much of the most well developed theory also applies strictly to a Hamiltonian system, neglecting forcing and dissipation processes that may maintain the flow in a quasi-equilibrium state. Some exceptions exist (e.g. Andrews, 1984a, b), but they are still quite rare.

\subsection{Equilibration and adjustment}

Linear stability theory generally applies to the behaviour of small-amplitude perturbations, but real flows are more typically observed when any incipient instabilities have grown to a large amplitude and are saturated. Precisely how equilibration is achieved may depend strongly on the structure of the flow and how it is maintained and/or dissipated. Even weakly nonlinear theories (e.g. Pedlosky, 1970, 1971; Drazin, 1970) show that growing instabilities act to reduce the vigour of the instability and its growth rate by modifying the structure of the initial background flow to make it less strongly unstable. For barotropically or baroclinically unstable flows, this commonly entails a combination of reducing the strength of any gradients of PV or buoyancy in the base state via a pattern of eddy fluxes, but the structure of the end state may depend on a variety of factors and constraints, including the strength and distribution of any forcing.

Where the forcing and dissipation is relatively weak, such that the characteristic timescale $\tau_{\mathrm{H}}$ for the system to respond to changes in forcing is much longer than any advective overturning timescale $\tau_{\mathrm{A}} \sim L / U$, fluid motions may be able to effectively nearly neutralise the instability so that, on timescales of $\mathrm{O}\left(\tau_{\mathrm{H}}\right)$ or longer, the flow stays close to a marginally unstable state. This scenario may offer the possibility of parameterising the effects of strong barotropic or 
baroclinic instabilities on large-scale flows through forms of barotropic or baroclinic adjustment (by analogy with convective adjustment). However, simply posing a requirement to seek a marginally unstable state is usually insufficient to determine or predict from first principles a unique equilibrated state unless additional constraints or information are available, such as an applicable optimisation principle (e.g. for entropy production, potential enstrophy or a generalised free energy; e.g. Lucarini, 2009; Hollerbach et al., 2004; Bouchet and Venaille, 2012).

Such an adjustment principle may have useful applicability to the interpretation of at least some observed flow systems, however. Dowling (1993) and Stamp and Dowling (1993), for example, have suggested that the zonalmean winds in the observable cloud level atmospheres of both Jupiter and Saturn may approach a state that is just marginally unstable with respect to a form of Arnol'd's second stability theorem, a hypothesis that seemed to be confirmed in more quantitative observations of potential vorticity (Read et al., 2006, 2009a). This was used by Read et al. (2009b) to infer the existence of a unique reference frame on each planet which represents the frame in which the gravest/deepest Rossby wave trains are just able to propagate at the same phase speed and thus couple together and interact via over-reflection. This frame corresponded closely on Jupiter to that of its magnetic field and deep interior. For Saturn, however, it differed significantly from the magnetic rotation periods inferred from measurements from the Voyager or Cassini spacecraft but agreed more closely with alternative estimates of Saturn's interior rotation from measurements of its gravity field or perturbations in its rings (Anderson and Schubert, 2007; Mankovitch et al., 2019).

\subsection{Baroclinic atmospheres}

For baroclinic atmospheres, such as that of Earth, the possibility persists that its zonal-mean circulation is at least partly controlled by a form of baroclinic adjustment (Stone, 1978), although debates continue to the present day as to whether this is a valid assumption and/or precisely what principle is being optimised during equilibration (e.g. Zurita-Gotor and Lindzen, 2007; Schneider, 2007). Flaws in Stone's original argument, that hinges on a tendency for the flow to deviate only slightly from a marginally unstable state, were discussed above, leading to alternative suggestions that the flow evolves towards a state with reduced nonlinear interactions (e.g. Schneider and Walker, 2006). Such a hypothesis is consistent with the observation that equilibrated flows in both the laboratory and in the atmospheres of Earth and some other planets exhibit the property that potential vorticity $Q \simeq Q(\psi)$.

The tendency shown in both laboratory experiments and simple GCM simulations for the total meridional heat transport (eddy plus zonal mean) to be largely independent of rotation rate (see Sects. 2.2 and 3) over the range in $B u$ and/or
$R o_{\mathrm{T}}$ from $\mathrm{O}(1)$ to $\sim 0.05$ is somewhat remarkable. It is apparently due to a mutual compensation between decreasing heat transport by the zonal-mean flow and increasing eddy heat transport with $\Omega$ until the latter reaches a peak around $R o_{\mathrm{T}} \simeq 0.1$. The resulting impact on the large-scale zonalmean flow is for the thermal structure to remain fairly static over this range of $\Omega$, which would imply that the mean slope of the isentropes in the latitude-height plane at mid-latitudes also remains largely independent of $\Omega$.

This is consistent with a roughly constant value of the criticality parameter,

$\xi=\frac{f}{\beta} \frac{\partial \bar{\theta} / \partial y}{\Delta \theta_{\mathrm{v}}}=-\frac{f}{\beta H} \frac{\partial \bar{\theta} / \partial y}{\partial \bar{\theta} / \partial z}$,

where $\Delta \theta_{\mathrm{v}}$ is a vertical contrast in potential temperature and $H$ is a vertical length scale, often taken to be the pressure scale height of an atmosphere. $\xi$ is thus a measure of the mean slope of the isentropes, which many studies have indicated remains close to $\mathrm{O}(1)$ over a wide range of parameters centred around those for Earth (e.g. Stone, 1978; Schneider and Walker, 2006; Zurita-Gotor and Lindzen, 2007). A number of different implications for $\xi \simeq 1$ have been noted, including an absence of a significant inverse kinetic energy cascade, since $\xi$ can also be interpreted (e.g. see Held and Larichev, 1996) as measuring the squared ratio of the Rhines scale,

$L_{\mathrm{R}}=\left(\frac{U}{\beta}\right)^{1 / 2}$,

which (based on the zonal-mean thermal wind scale $U \sim U_{\mathrm{T}}$ ) can estimate the large-scale limit to the main inverse cascade to the Rossby deformation radius $L_{\mathrm{D}}$ (although $L_{\mathrm{R}}$ is more conventionally estimated using $U \sim(E K E)^{1 / 2}$ which can differ significantly from $U_{\mathrm{T}}$ under some conditions). This would also suggest a suppression of nonlinear wave-wave interactions, which would be consistent with equilibration towards a state in which $\boldsymbol{u} \cdot \nabla Q \simeq 0$, implying that $Q \simeq Q(\psi)$ in a quasi-geostrophic flow (where $\psi$ is the horizontal stream function). Something close to this configuration of $Q$ and $\psi$ occurs within the regular baroclinic wave regime in rotating annulus experiments (e.g. Read et al., 1986), and even Earth's atmosphere seems to approach such a state to some extent (e.g. Butchart et al., 1989).

Recent work (e.g. Vallis, 1988; Zurita-Gotor, 2008; Jansen and Ferrari, 2012) has questioned whether the preferred value of $\xi$ is universally around $\mathrm{O}(1)$ for all situations, noting that conditions in the oceans, for example, may favour equilibrated values of $\xi \gg 1$ (Jansen and Ferrari, 2012, 2013). Jansen and Ferrari (2013) suggest that the equilibrated value of $\xi$ in a baroclinic atmosphere is likely to be set by a balance between differential heating through radiative forcing (with radiative adjustment timescale $\tau_{\mathrm{R}}$ ) and eddy heat fluxes, parameterised via a diffusivity (for PV) $D$. This leads to an estimate of the isentropic slope $s \sim H / \sqrt{\tau_{\mathrm{R}} D}$ along a length 
scale $\ell_{\text {diff }} \sim \sqrt{\tau_{R} \mathrm{D}}$, over which eddies can transport PV and heat within the radiative timescale $\tau_{\mathrm{R}}$ and thus

$\xi \sim \frac{s a}{H} \sim \frac{a}{\sqrt{\tau_{\mathrm{R}} D}}$

(e.g. Jansen and Ferrari, 2012, 2013). If $\ell_{\text {diff }} \ll a$, therefore, values of $\xi \gg 1$ may occur, whereas if $\ell_{\text {diff }} \gtrsim a$, then heat transfer by eddies is efficient enough to homogenise potential temperature horizontally, relaxing the isentropic slope such that $\xi \leq 1$.

Taking a broader view across a wide range of parameter space and different physical systems, however, the discussion above in Sects. 2-3 suggests that a more complete explanation for many of the recently published results on criticality and baroclinic adjustment may be related to the extent to which variations in heat transports, not only due to eddies but also to the zonal-mean circulation, may mutually compensate each other as parameters are varied so that the total heat transport is maintained at a nearly constant rate. This compensation may be most effective within the typical range $0.05<R o_{\mathrm{T}} \leq 1$, within which eddy heat transports increase with decreasing $R o_{\mathrm{T}}$, while zonal-mean transport decreases. Beyond this limit (i.e. $R o_{\mathrm{T}}<0.05$ ), both components of heat transport decrease as $R o_{\mathrm{T}}$ decreases, and the zonal-mean thermal structure might be expected to relax gradually towards the radiative-convective equilibrium state as the transport by the zonal-mean circulation becomes small compared to the eddy transport and the eddy diffusivity $D$ decreases. The very high rotation rate limit of $\xi$ would then depend upon the slope of the isentropes in this radiative-convective equilibrium state but could well lie close to $\mathrm{O}(1)$ for Earth-like atmospheric conditions. For other systems, however, such as in Earth's oceans, the criticality $\xi$ has been suggested to be $\gg 1$ (e.g. Vallis, 1988; Jansen and Ferrari, 2012), and a similar criticality may well apply in the atmospheres of the gas giants (Jupiter and Saturn), where $D$ is likely very small and the natural slope of isentropes in the convectively active troposphere is very steep. The existence or otherwise of a genuine baroclinic adjustment regime, however, depends upon the existence of a range of parameters over which eddy heat transports strengthen with $\Omega$ and so can compensate for changes in the zonal-mean transport. While this seems to be plausible for Earth-like atmospheres, it may not be completely general and should be investigated more thoroughly in future work.

\subsection{Planetary atmospheres}

The possible existence of both baroclinic and barotropic adjustment regimes immediately raises the question as to whether any of the known planetary atmosphere circulations are actually in one of these regimes. Given the limits on $R_{\mathrm{T}}$ for the baroclinic adjustment regime as described here, Earth itself would seem to lie close to the high $\Omega$ margins of this regime, which would be consistent with recent work highlighting its possible effects on the circulation.
Mars, with a value of $R o_{\mathrm{T}} \simeq 0.2$ (see Table 2), would also appear at first sight to be firmly within the baroclinic adjustment regime. However, it might not fulfil the other condition for the existence of a baroclinic adjustment regime, namely, that forcing timescales $\tau_{\mathrm{S}} \gg \tau_{\mathrm{A}}$. Radiative adjustment timescales on Mars are typically only around $\tau_{\mathrm{R}} \simeq 1 \mathrm{~d}$, which is comparable to $\tau_{\mathrm{A}}=L / U$ based on typical horizontal velocities and the planetary radius. Radiative forcing is therefore relatively much stronger on Mars than on Earth, which might suggest from Eq. (18) that baroclinic adjustment would lead to an adjusted state with $\xi>1$. Under these strongly forced/damped conditions, however, flow configurations that appear to be vigorously unstable, based on criteria derived for non-dissipative flows, may actually be relatively stable in practice. This was illustrated recently in the work of Seviour et al. (2017), for example, who noted that zonalmean PV at high latitudes on Mars often took the form of a persistent annular band, across which were strong reversals in the sign of $\partial Q / \partial y$. Seviour et al. (2017) were able to reproduce this behaviour in a simple model in which zonalmean forcing was applied by relaxing to a similar PV distribution with a short relaxation timescale. Baroclinic adjustment concepts are therefore unlikely to apply to Mars in any useful sense.

The cases of slowly rotating planets, such as Venus or Titan in the solar system, would also seem to place them outside of the limits on $R_{\mathrm{T}}$ for baroclinic adjustment to be effective, since heat transports are then dominated by the zonal-mean circulation and not by eddies. Their largescale thermal structure is typically characterised by very weak horizontal thermal gradients compared to their stratification, so isentropic slopes are very small. This would indicate that $\xi \ll 1$ with relatively weak eddy heat transports, at least on the planetary scale. The strong super-rotation of the atmospheres of Venus (and possibly Titan), however, seems to allow for a role for baroclinic instabilities in atmospheric layers that rotate fast enough to be in a quasigeostrophic regime, with local values of $R o_{\mathrm{T}} \sim 0.1$ (see Table 2). Radiative-relaxation timescales near the cloud tops of Venus are also relatively long compared with advective overturning timescales, so baroclinic adjustment effects might be relevant to the circumpolar regions of Venus's cloudy layers, a possibility that deserves further investigation.

Jupiter would seem to be well outside its baroclinic adjustment regime, based simply on its values of $R o_{\mathrm{T}}$ and $\mathrm{Bu}$ (see Table 2). Recent work has demonstrated that Jupiter exhibits a substantial upscale cascade of kinetic energy at its cloud tops, both spectrally local and non-local (Young and Read, 2017). This is consistent with estimates that suggest that $L_{\mathrm{R}}$ is significantly larger than $L_{\mathrm{D}}$ on Jupiter (Galperin et al., 2014), indicating that Jupiter's weather layer is significantly super-critical with an effective value for $\xi$ of around 5. This would seem to be because the relevant scale for $U$ in $L_{\mathrm{R}}$ is much larger than the thermal wind scale $U_{\mathrm{T}}$, with much of the kinetic energy at Jupiter's cloud tops residing in 
its zonal jets. Such jets may well be predominantly barotropic in structure, at least within the weather layer.

It is perhaps unsurprising, therefore, that barotropic adjustment seems to be a useful concept in the context of the gas giant planets, Jupiter and Saturn, as discussed above, with the circulation in the weather layers remaining close to a marginally unstable state, as determined by the Arnol'd II criterion. It seems reasonable that the weather layers of Uranus and Neptune might also be in a similar regime, which could readily be investigated in both observations and models given the availability of suitable data. The quality and coverage of the original data from the Voyager encounter may not be adequate to verify these ideas by themselves, although the accuracy and latitude coverage of cloud motion tracking has improved significantly in recent years from Earthbased (ground and space) telescopes (e.g. Garcia-Melendo and Sánchez-Lavega, 2001). These issues should certainly be addressed in the context of future missions currently being planned for the ice giants.

In the context of the ever-expanding discoveries of extrasolar planets, it seems highly likely that many of them will host atmospheres within which baroclinic or barotropic instabilities may play an active role. Future investigations of their possible climates and atmospheric circulations will therefore need to take fairly full account of the influence of these instabilities, for which equilibration and adjustment concepts may surely prove useful in at least some cases.

Data availability. Data on the Martian atmosphere can be obtained from the website of the Mars Climate Database (Lewis et al., 1999; Millour et al., 2018), via the graphical interface http://www-mars.lmd.jussieu.fr/mcd_python/ (last access: 30 March 2020). Data from the model simulations of Young et al. (2019a) can be obtained from Oxford University's Research Archive at https://doi.org/10.5287/bodleian:PyYbbxpk2 (Young et al., 2018). Data from laboratory experiments reported in Sect. 2 may be obtainable from the original authors on request.

Author contributions. PR supervised much of the research reported from the University of Oxford, analysed some of the laboratory data and was the lead author of the paper. NL, FTV, YW and RY carried out computations and analysis for model simulations, and DK computed the energy budget reported in Sect. 4.3. Other authors also contributed to writing the text.

Competing interests. The authors declare that they have no conflict of interest.

Special issue statement. This article is part of the special issue "Centennial issue on nonlinear geophysics: accomplishments of the past, challenges of the future". It is not associated with a conference.
Acknowledgements. This article originated from a lecture prepared by PLR for the Summer School on Waves, Instability and Turbulence in Geophysical and Astrophysical Flows in Cargèse, Corsica, in July 2019. We are grateful to Geoff Vallis and an anonymous referee for their comments on an earlier version of this paper.

Financial support. Peter Read, Neil Lewis, Fachreddin TabatabaVakili and Roland Young have been supported by the UK Science and Technology Research Council during the course of this research (grant nos. ST/K502236/1, ST/K00106X/1 and ST/I001948/1). Hélène Scolan and Peter Read have been supported by the UK Engineering and Physics Sciences Research Council (grant no. EP/K029428/1). Daniel Kennedy was supported via an internship from the UK Met Office Academic Partnership, and Susie Wright was supported by a research studentship from the UK Natural Environment Research Council and the UK Met Office.

Review statement. This paper was edited by Olivier Talagrand and reviewed by Geoff Vallis and one anonymous referee.

\section{References}

Aguiar, A. C. B., Read, P. L., Wordsworth, R. D., Salter, T., and Yamazaki, Y. H.: A laboratory model of Saturn's North Polar Hexagon, Icarus, 206, 755-763, 2010.

Anderson, J. D. and Schubert, G.: Saturn's gravitational field, internal rotation, and interior structure, Science, 317, 1384-1387, 2007.

Andrews, D. G.: On the existence of nonzonal flows satisfying sufficient conditions for stability, Geophys. Astrophys. Fluid Dyn., 28, 243-256, 1984a.

Andrews, D. G.: On the stability of forced non-zonal flows, Q. J. Roy. Meteor. Soc., 110, 657-662, 1984b.

Antuñano, A., del Río-Gaztelurrutia, T. and. Sánchez-Lavega, A., Read, P. L., and Fletcher, L. N.: Potential Vorticity of Saturn's Polar Regions: Seasonality and Instabilities, J. Geophys. Res., 124, 186-201, https://doi.org/10.1029/2018JE005764, 2019.

Arnol'd, V. I.: On an a priori estimate in the theory of hydrodynamical stability, Izv. Vyssh. Ucheb. Zaved. Matematika, 54, 3-5, 1966 (in Russian).

Barnes, J. R.: Linear baroclinic instability in the Martian atmosphere, J. Atmos. Sci., 41, 1536-1550, 1984.

Bell, M. J. and White, A. A.: The stability of internal baroclinic jets: some analytical results, J. Atmos. Sci., 45, 2571-2590, 1988.

Boer, G. J. and Lambert, S.: The energy cycle in atmospheric models, Clim. Dyn., 30, 371-390, 2008.

Bouchet, F. and Venaille, A.: Statistical mechanics of twodimensional and geophysical flows, Phys. Rep., 515, 227-295, https://doi.org/10.1016/j.physrep.2012.02.001, 2012.

Bowden, M.: An experimental investigation of heat transfer in rotating fluids, PhD thesis, Durham University, UK, 1961.

Branscombe, L. E.: The Charney Baroclinic Instability Problem: Approximate Solutions and Modal Structures, J. Atmos. Sci., 40, 1393-1409, 1983. 
Butchart, N., Haines, K., and Marshall, J. C.: A theoretical and diagnostic study of solitary waves and atmospheric blocking, J. Atmos. Sci., 46, 2063-2078, 1989.

Charney, J. G. and Stern, M. E.: On the Stability of Internal Baroclinic Jets in a Rotating Atmosphere, J. Atmos. Sci., 19, 159-172, 1962.

Conrath, B. J., Gierasch, P. J., and Nath, N.: Stability of zonal flows on Jupiter, Icarus, 48, 256-282, 1981.

Dowling, T.: A relationship between potential vorticity and zonal wind on Jupiter, J. Atmos. Sci., 50, 14-22, 1993.

Dowling, T.: Dynamics of Jovian atmospheres, Ann. Rev. Fluid Mech., 27, 293-334, 1995.

Drazin, P. G.: Non-linear baroclinic instability of a continuous zonal flow, Q. J. Roy. Meteor. Soc., 96, 667-676, 1970.

Eady, E. T.: Long Waves and Cyclone Waves, Tellus, 1, 33-52, 1949.

Flór, J.-B., Scolan, H., and Gula, J.: Frontal instabilities and waves in a differentially rotating fluid, J. Fluid Mech., 685, 532-542, https://doi.org/10.1017/jfm.2011.338, 2011.

Fraedrich, K., Kirk, E., Luksch, U., and Lunkeit, F.: The Portable University Model of the Atmosphere (PUMA): Storm track dynamics and low frequency variability, Meteorol. Zeitschrift, 14, 735-745, 2005.

Frisius, T., Lunkeit, F., Fraedrich, K., and James, I. N.: Stormtrack organization and variability in a simplified global circulation model, Q. J. Roy. Meteor. Soc., 124, 1019-1043, 1998.

Früh, W.-G. and Read, P. L.: Experiments on a barotropic rotating shear layer. Part 1. Instability and steady vortices, J. Fluid Mech., 383, 143-173, 1999a.

Früh, W.-G. and Read, P. L.: Flow field and point velocity measurements in a barotropically unstable shear layer, Phys. Chem. Earth (B), 24, 461-466, 1999 b.

Galperin, B., Young, R., Sukoriansky, S., Dikovskaya, N., Read, P., Lancaster, A., and Armstrong, D.: Cassini observations reveal a regime of zonostrophic macroturbulence on Jupiter, Icarus, 229, 295-320, 2014.

Garcia-Melendo, E. and Sánchez-Lavega, A.: A study of the stability of Jovian zonal winds from HST images: 1995-2000, Icarus, 152, 316-330, 2001.

Geisler, J., Pitcher, E., and Malone, R.: Rotating-Fluid experiments with an atmospheric general circulation model, J. Geophys. Res., 88, 9706-9716, https://doi.org/10.1029/JC088iC14p09706, 1983.

Gierasch, P. J., Ingersoll, A. P., and Pollard, D.: Baroclinic instabilities in Jupiter's zonal flow, Icarus, 40, 205-212, 1979a.

Gierasch, P. J., Thomas, P., French, R., and Veverka, J.: Spiral clouds on Mars: a new artmospheric phenomenon, Geophys. Res. Lett., 6, 405-408, 1979b.

Gierasch, P. J., Ingersoll., A. P., Banfield, D., Ewald, S. P., Helfenstein, P., Simon-Miller, A., Vasavada, A., Breneman, H. H., Senske, D. A., and the Galileo Imaging Team: Observation of moist convection in Jupiter's atmosphere, Nature, 403, 628-630, 2000

Guillot, T.: The Interiors of Giant Planets: Models and Outstanding Questions, Ann. Rev. Earth Planet Sci., 33, 493-530, https://doi.org/10.1146/annurev.earth.32.101802.120325, 2005.

Gula, J., Plougonven, R., and Zeitlin, V.: Ageostrophic instabilities of fronts in a channel in a stratified rotating fluid, J. Fluid Mech.,
627, 485-507, https://doi.org/10.1017/S0022112009006508, 2009.

Hayashi, Y.-Y. and Young, W. R.: Stable and unstable shear modes of rotating parallel flows in shallow water, J. Fluid Mech., 184, 477-504, 1997.

Held, I. M. and Larichev, V. D.: A scaling theory for horizontally homogeneous baroclinically unstable flow on a beta plane, J. Atmos. Sci., 53, 946-952, 1996.

Hide, R.: Some laboratory experiments on free thermal convection in a rotating fluid subject to a horizontal temperature gradient and their relation to the theory of the global atmospheric circulation, in: The Global Circulation of the Atmosphere, edited by: Corby, G. A., pp. 196-221, Royal Meteorological Society, London, 1969.

Hide, R. and Mason, P. J.: Sloping convection in a rotating fluid, Adv. Phys., 24, 47-100, 1975.

Hide, R. and Titman, C. W.: Detached shear layers in a rotating fluid, J. Fluid Mech., 29, 39-60, 1967.

Hollerbach, R., Futterer, B., More, T., and Egbers, C.: Instabilities of the Stewartson layer Part 2. supercritical mode transitions, Theoret. Comput. Fluid Dyn., 19, 197-204, https://doi.org/10.1007/s00162-004-0125-5, 2004.

Hunt, B. G.: The influence of the Earth's rotation rate on the general circulation of the atmosphere, J. Atmos. Sci., 36, 1392-1408, 1979.

Ingersoll, A., Gierasch, P., Banfield, D., and Vasavada, A.: Moist convection as an energy source for the large-scale motions in Jupiter's atmosphere. Galileo Imaging Team, Nature, 403, 630 2, https://doi.org/10.1038/35001021, 2000.

Ingersoll, A. P. and Cuzzi, J. N.: Dynamics of Jupiter's cloud bands, J. Atmos. Sci., 26, 981-985, 1969.

Ingersoll, A. P., Beebe, R. F., Mitchell, J. L., Garneau, G. W., Yagi, G. M., and Müller, J.-P.: Interaction of eddies and mean zonal flow on Jupiter as inferred from Voyager 1 and 2 images, J. Geophys. Res., 86, 8733-8743, 1981.

James, I. N.: Introduction to Circulating Atmospheres, Cambridge University Press, Cambridge, UK, 1994.

Jansen, M. and Ferrari, R.: Macroturbulent Equilibration in a Thermally Forced Primitive Equation System, J. Phys. Oceanogr., 69 695-713, 2012.

Jansen, M. and Ferrari, R.: Equilibration of an Atmosphere by Adiabatic Eddy Fluxes, J. Atmos. Sci., 70, 2948-2962, 2013.

Kaspi, Y. and Showman, A. P.: Atmospheric dynamics of terrestrial exoplanets over a wide range of orbital and atmospheric parameters, Astrophys. J., 804, 60, https://doi.org/10.1088/0004637X/804/1/60, 2015.

Kaspi, Y., Galanti, E., Hubbard, W. B., Stevenson, D. J., Bolton, S. J., Iess, L., Guillot, T., Bloxham, J., Connerney, J. E. P., Cao, H., Durante, D., Folkner, W. M., Helled, R., Ingersoll, A. P., Levin, S. M., Lunine, J. I., Miguel, Y., Militzer, B., Parisi, M., and Wahl, S. M.: Jupiter's atmospheric jet streams extend thousands of kilometres deep, Nature, 555, 223-226, https://doi.org/10.1038/nature25793, 2018.

Kong, D., Zhang, K., Schubert, G., and Anderson, J. D.: Origin of Jupiter's cloud-level zonal winds remains a puzzle even after Juno, P. Natl. Acad. Sci., 115, 8499-8504, https://doi.org/10.1073/pnas.1805927115, 2018.

Lebonnois, S., Hourdin, F., Eymet, V., Crespin, A., Fournier, R., and Forget, F.: Superrotation of Venus' atmosphere analyzed with a 
full general circulation model, J. Geophys. Res., 115, E06006, https://doi.org/10.1029/2009JE003458, 2010.

Lebonnois, S., Lee, C., Yamamoto, M., Dawson, J., Lewis, S. R., Mendonca, J., Read, P., Parish, H. F., Schubert, G., Bengtsson, L., Grinspoon, D., Limaye, S. S., Schmidt, H., Svedhem, H., and Titov, D. V.: Models of Venus' atmosphere, in: Towards Understanding the Climate of Venus: Applications of Terrestrial Models to Our Sister Planet, edited by: Bengtsson, L., Bonnet, R.-M., Grinspoon, D., Koumoutsaris, S., Lebonnois, S., and Titov, D., Springer, New York, 129-156, 2013.

Lebonnois, S., Sugimoto, N., and Gilli, G.: Wave analysis in the atmosphere of Venus below $100-\mathrm{km}$ altitude, simulated by the LMD Venus GCM, Icarus, 278, 38-51, https://doi.org/10.1016/j.icarus.2016.06.004, 2016.

Lee, C. and Richardson, M. I.: A general circulation model ensemble study of the atmospheric circulation of Venus, J. Geophys. Res., 115, E04002, https://doi.org/10.1029/2009JE003490, 2010.

Lewis, G. M. and Nagata, W.: Linear stability analysis for the differentially heated rotating annulus, Geophys. Astrophys. Fluid Dyn., 98, 129-152, 2004.

Lewis, G. M., Périnet, N., and van Veen, L.: Primary flow transitions in the baroclinic annulus: Prandtl number effects, in: Modelling Atmospheric and Oceanic Flows: Insights from Laboratory Experiments and Numerical Simulations, edited by: von Larcher, T. and Williams, P. D., American Geophysical Union and Wiley, Hoboken, NJ, USA, 45-60, 2015.

Lewis, S. R., Collins, M., Read, P. L., Forget, F., Hourdin, F., Fournier, R., Hourdin, C., Talagrand, O., and Huot, J.-P.: A Climate Database for Mars, J. Geophys. Res., 104, 24177-24194, 1999.

Lewis, S. R., Dawson, J., Lebonnois, S., and Yamamoto, M.: Modeling efforts, in: Towards Understanding the Climate of Venus: Applications of Terrestrial Models to Our Sister Planet, edited by: Bengtsson, L., Bonnet, R.-M., Grinspoon, D., Koumoutsaris, S., Lebonnois, S., and Titov, D., pp. 102-128, Springer, New York, 2013.

Lewis, S. R., Mulholland, D. P., Read, P. L., Montabone, L., Wilson, R. J., and Smith, M. D.: The solsticial pause on Mars: 1. A planetary wave reanalysis, Icarus, 264, 456-464, https://doi.org/10.1016/j.icarus.2015.08.039, 2016.

Lian, Y. and Showman, A. P.: Deep jets on gas-giant planets, Icarus, 194, 597-615, https://doi.org/10.1016/j.icarus.2007.10.014, 2008.

Lian, Y. and Showman, A. P.: Generation of equatorial jets by largescale latent heating on the giant planets, Icarus, 207, 373-393, https://doi.org/10.1016/j.icarus.2009.10.006, 2010.

Lindzen, R. S.: Instability of a plane-parallel shear flow (Toward a mechanism for how it works), PAGEOPH, 126, 103-121, 1988.

Liu, J. and Schneider, T.: Mechanisms of Jet Formation on the Giant Planets, J. Atmos. Sci., 67, 3652-3672, https://doi.org/10.1175/2010JAS3492.1, 2010.

Lorenz, E. N.: Available Potential Energy and the Maintenance of the General Circulation, Tellus, 7, 157-167, 1955.

Lorenz, E. N.: The Nature and Theory of the General Circulation of the Atmosphere, WMO, No. 218, T. P. 115, Geneva, Switzerland, 1967.
Lucarini, V.: Thermodynamic efficiency and entropy production in the climate system, Phys. Rev. E, 80, 021118, https://doi.org/10.1103/PhysRevE.80.021118, 2009.

Mankovitch, C., Marley, M. S., Fortney, J. J., and Movshovitz, N.: Cassini ring seismology as a probe of Saturn's interior. I. rigid rotation, Astrophys. J., 871, 1, https://doi.org/10.3847/15384357/aaf798, 2019.

Margules, M.: Über die Energie der Stürme, Jahrb. Kais.-kön Zent. für Met. und Geodynamik, Vienna, pp. 1-26, 1903.

Mendonça, J. M. and Read, P. L.: Exploring the Venus global super-rotation using a comprehensive general circulation model, Planet. Space Sci., 134, 1-18, 2016.

Merlis, T. M. and Schneider, T.: Atmospheric dynamics of Earthlike tidally locked aquaplanets, J. Adv. Model. Earth Syst., 2, 13, https://doi.org/10.3894/JAMES.2010.2.13, 2010.

Millour, E., Forget, F., Spiga, A., Vals, M., Zakharov, V., Montabone, L., Lefèvre, F., Montmessin, F., Chaufray, J.-Y., López-Valverde, M. A., González-Galindo, F., Lewis, S. R., Read, P. L., Desjean, M.-C., Cipriani, F., and the MCD development team: The Mars Climate Database (Version 5.3), Proceedings of Scientific Workshop: "From Mars Express to ExoMars”, 27-28 February 2018, European Space Astronomy Centre (ESAC), Madrid, Spain, 2018.

Mitchell, J. L. and Vallis, G. K.: The transition to superrotation in terrestrial atmospheres, J. Geophys. Res., 115, E12008, https://doi.org/10.1029/2010JE003587, 2010.

Montabone, L., Marsh, K., Lewis, S. R., Read, P. L., Smith, M. D., Holmes, J., Spiga, A., Lowe, D., and Pamment, A.: The Mars Analysis Correction Data Assimilation (MACDA) Dataset V1.0, Geosci. Data J., 1, 129-139, https://doi.org/10.1002/gdj3.13, 2014.

Mu, M. and Wu, Y.-H.: Arnol'd nonlinear stability theorems and their application to the atmosphere and oceans, Surv. Geophys., 22, 383-426, 2001.

Niino, H. and Misawa, N.: An experimental and theoretical study of barotropic instability, J. Atmos. Sci., 41, 1992-2011, 1984.

Palotai, C., Dowling, T. E., and Fletcher, L. N.: 3D Modeling of interactions between Jupiter's ammonia clouds and large anticyclones, Icarus, 232, 141-156, https://doi.org/10.1016/j.icarus.2014.01.005, 2014.

Pedlosky, J.: Finite-amplitude baroclinic waves, J. Atmos. Sci., 27, 15-30, 1970.

Pedlosky, J.: Finite-amplitude baroclinic waves with small dissipation, J. Atmos. Sci., 28, 587-597, 1971.

Peixoto, J. and Oort, A.: The Annual Distribution of Atmospheric Energy on a Planetary Scale, J. Geophys. Res., 20, 2149-2159, https://doi.org/10.1029/JC079i015p02149, 1974.

Read, P. L.: A combined laboratory and numerical study of heat transport by baroclinic eddies and axisymmetric flows, J. Fluid Mech., 489, 301-323, 2003.

Read, P. L.: Dynamics and circulation regimes of terrestrial planets, Plan. Space Sci., 59, 900-914, 2011.

Read, P. L. and Lebonnois, S.: Superrotation on Venus, on Titan, and Elsewhere, Ann. Rev. Earth Planet. Sci., 46, 175-202, https://doi.org/10.1146/annurev-earth-082517-010137, 2018.

Read, P. L., Rhines, P. B., and White, A. A.: Geostrophic scatter diagrams and potential vorticity dynamics, J. Atmos. Sci., 43, 3226-3240, 1986. 
Read, P. L., Gierasch, P. J., Conrath, B. J., Simon-Miller, A., Fouchet, T., and Yamazaki, Y. H.: Mapping potential-vorticity dynamics on Jupiter. I: Zonal-mean circulation from Cassini and Voyager 1 data, Q. J. Roy. Meteor. Soc., 132, 1577-1603, https://doi.org/10.1256/qj.05.34, 2006.

Read, P. L., Conrath, B., Fletcher, L., Gierasch, P., SimonMiller, A., and Zuchowski, L.: Mapping potential-vorticity dynamics on Saturn: Zonal-mean circulation from Cassini and Voyager data, Planet. Space Sci., 57, 1682-1698, https://doi.org/10.1016/j.pss.2009.03.004, 2009a.

Read, P. L., Dowling, T. E., and Schubert, G.: Saturn's rotation period from its planetary wave configuration, Nature, 460, 608610, https://doi.org/10.1038/nature08194, 2009b.

Read, P. L., Pérez, E. P., Moroz, I. M., and Young, R. M. B.: General Circulation of Planetary Atmospheres: Insights from Rotating Annulus and Related Experiments, in: Modelling Atmospheric and Oceanic Flows: Insights from Laboratory Experiments and Numerical Simulations, edited by: von Larcher, T. and Williams, P. D., American Geophysical Union and Wiley, Hoboken, NJ, USA, pp. 9-44, 2015.

Read, P. L., Tabataba-Vakili, F., Wang, Y., Augier, P., Lindborg, E., Valeanu, A., and Young, R. M. B.: Comparative terrestrial atmospheric circulation regimes in simplified global circulation models. Part II: Energy budgets and spectral transfers, Q. J. Roy. Meteor. Soc., 144, 2558-2576, https://doi.org/10.1002/qj.3351, 2018.

Ripa, P.: General stability conditions for zonal flows in a one-layer model on the $\beta$-plane or the sphere, J. Fluid Mech., 126, 463489, 1983.

Sakai, S.: Rossby-Kelvin instability: a new type of ageostrophic instability caused by a resonance between Rossby waves and gravity waves, J. Fluid Mech., 202, 149-176, 1989.

Salyk, C., Ingersoll, A. P., Lorre, J., Vasavada, A., and Del Genio, A. D.: Interaction between eddies and mean flow in Jupiter's atmosphere: analysis of Cassini imaging data, Icarus, 185, 430442, https://doi.org/10.1016/j.icarus.2006.08.007, 2006.

Sánchez-Lavega, A., Lebonnois, S., Imamura, T., Read, P., and Luz, D.: The Atmospheric Dynamics of Venus, Space Sci. Rev., 212, 1541-1616, https://doi.org/10.1007/s11214-017-0389-x, 2017.

Schneider, T.: The thermal stratification of the extratropical troposphere, in: The Global Circulation of the Atmosphere, edited by: Schneider, T. and Sobel, A. H., pp. 47-77, Princeton University Press, Princeton, USA, 2007.

Schneider, T. and Walker, C. C.: Self-organization of atmospheric macroturbulence into critical states of weak nonlinear eddy-eddy interactions, J. Atmos. Sci., 63, 1569-1586, 2006.

Scott, R. K. and Dunkerton, T. J.: Vertical structure of tropospheric winds on gas giants, Geophys. Res. Lett., 44, 3073-3081, https://doi.org/10.1002/2017GL072628, 2017.

Seviour, W. J. M., Waugh, D. W., and Scott, R. K.: The stability of Mars's annular polar vortex, J. Atmos. Sci., 74, 1533-1547, https://doi.org/10.1175/JAS-D-16-0293.1, 2017.

Sommeria, J., Meyers, S. D., and Swinney, H. L.: Experiments on vortices and Rossby waves in eastward and westward jets, in: Nonlinear Topics in Ocean Physics, edited by: Osborne, A. R., pp. 227-269, North Holland, Amsterdam, 1991.
Spiga, A., Guerlet, S., Millour, E., Indurain, M., Meurdesoif, Y., Cabanes, S., Dubos, T., Leconte, J., Boissinot, A., Lebonnois, S., Sylvestre, M., and Fouchet, T.: Global climate modeling of Saturn's atmosphere. Part II: Multi-annual high-resolution dynamical simulations, Icarus, 335, 113377, https://doi.org/10.1016/j.icarus.2019.07.011, 2020.

Stamp, A. P. and Dowling, T.: Jupiter's winds and Arnol'd's second stability theorem: slowly moving waves and neutral stability, J. Geophys. Res., 98, 18847-18855, 1993.

Stone, P.: Baroclinic Adjustment., J. Atmos. Sci., 35, 561-571, https://doi.org/10.1175/15200469(1978)035<0561:BA>2.0.CO;2, 1978.

Sugimoto, N., Takagi, M., and Matsuda, Y.: Baroclinic instability in the Venus atmosphere simulated by GCM, J. Geophys. Res., 119, 1950-1968, https://doi.org/10.1002/2014JE004624, 2014.

Tabataba-Vakili, F., Read, P. L., Lewis, S. R., Montabone, L., Ruan, T., Wang, Y., Valeanu, A., and Young, R. M. B.: A Lorenz/Boer energy budget for the atmosphere of Mars from a "reanalysis" of spacecraft observations, Geophys. Res. Lett., 42, 8320-8327, https://doi.org/10.1002/2015GL065659, 2015.

Vallis, G. K.: Numerical studies of eddy transport properties in eddy-resolving and parametrized models, Q. J. Roy. Meteor. Soc., 114, 183-204, 1988.

Vallis, G. K.: Atmospheric and Oceanic Fluid Dynamics - Fundamentals and Large-Scale Circulation (2nd Edition), Cambridge University Press, Cambridge, UK, 2017.

Wang, Y., Read, P. L., Tabataba-Vakili, F., and Young, R. M. B.: Comparative terrestrial atmospheric circulation regimes in simplified global circulation models. Part I: From cyclostrophic super-rotation to geostrophic turbulence, Q. J. Roy. Meteor. Soc., 144, 2537-2557, https://doi.org/10.1002/qj.3350, 2018.

Williams, G. and Holloway, J.: The range and unity of planetary circulations, Nature, 297, 295-299, 1982.

Young, R., Read, P., and Wang, Y.: Simulating Jupiter's weather layer: Accompanying data for Parts I and II, University of Oxford, https://doi.org/10.5287/bodleian:PyYbbxpk2, 2018.

Young, R. E., Houben, H., and Pfister, L.: Baroclinic instability in the Venus atmosphere, J. Atmos. Sci., 41, 2310-2333, 1984.

Young, R. M. B.: The Lorenz energy cycle in simulated rotating annulus flows, Phys. Fluids, 26, 056602, https://doi.org/10.1063/1.4873921, 2014.

Young, R. M. B. and Read, P. L.: Forward and inverse kinetic energy cascades in Jupiter's turbulent weather layer, Nature Phys., 13, 1135-1140, https://doi.org/10.1038/NPHYS4227, 2017.

Young, R. M. B., Read, P. L., and Wang, Y.: Simulating Jupiter's weather layer. Part I: Jet spin-up in a dry atmosphere, Icarus, 326, 225-252, 2019a.

Young, R. M. B., Read, P. L., and Wang, Y.: Simulating Jupiter's weather layer. Part II: Passive ammonia and water cycles, Icarus, 326, 253-268, 2019b.

Zurita-Gotor, P.: The sensitivity of the isentropic slope in a primitive equation dry model, J. Atmos. Sci., 65, 43-65, 2008.

Zurita-Gotor, P. and Lindzen, R. S.: Theories of baroclinic adjustment and eddy equilibrations, in: The Global Circulation of the Atmosphere, edited by: Schneider, T. and Sobel, A. H., pp. 2246, Princeton University Press, Princeton, USA, 2007. 